\title{
Nonideal Sampling and Regularization Theory
}

\author{
Sathish Ramani, Dimitri Van De Ville, Member, IEEE, Thierry Blu, Senior Member, IEEE, and \\ Michael Unser, Fellow, IEEE
}

\begin{abstract}
Shannon's sampling theory and its variants provide effective solutions to the problem of reconstructing a signal from its samples in some "shift-invariant" space, which may or may not be bandlimited. In this paper, we present some further justification for this type of representation, while addressing the issue of the specification of the best reconstruction space. We consider a realistic setting where a multidimensional signal is prefiltered prior to sampling, and the samples are corrupted by additive noise. We adopt a variational approach to the reconstruction problem and minimize a data fidelity term subject to a Tikhonov-like (continuous domain) $L_{2}$-regularization to obtain the continuous-space solution. We present theoretical justification for the minimization of this cost functional and show that the globally minimal continuous-space solution belongs to a shift-invariant space generated by a function (generalized B-spline) that is generally not bandlimited. When the sampling is ideal, we recover some of the classical smoothing spline estimators. The optimal reconstruction space is characterized by a condition that links the generating function to the regularization operator and implies the existence of a B-spline-like basis. To make the scheme practical, we specify the generating functions corresponding to the most popular families of regularization operators (derivatives, iterated Laplacian), as well as a new, generalized one that leads to a new brand of Matérn splines. We conclude the paper by proposing a stochastic interpretation of the reconstruction algorithm and establishing an equivalence with the minimax and minimum mean square error (MMSE/Wiener) solutions of the generalized sampling problem.
\end{abstract}

Index Terms-Matérn class, minimax and minimum mean square error (MMSE) reconstruction, nonideal sampling, regularization, shift-invariant spaces, smoothing splines, Tikhonov criterion, Wiener solution.

\section{INTRODUCTION}

$\mathbf{S}$ HANNON's sampling theory provides an elegant method to perfectly reconstruct bandlimited signals from their equidistant samples [1]-[5]. Mathematically, Shannon's sampling/reconstruction process for 1-D bandlimited functions is equivalent to projecting the input signal $f(x)$ onto the shift-invariant space $V=\operatorname{span}\{\operatorname{sinc}(x-k)\}_{k \in \mathbb{Z}}$, spanned by the integer-shifts ${ }^{1}$ of the $\operatorname{sinc}-f u n c t i o n \operatorname{sinc}(x)$ [6]-[9]. There are extensions of the classical sampling theorem for

Manuscript received November 28, 2006; revised August 19, 2007. The associate editor coordinating the review of this manuscript and approving it for publication was Dr. Yonina C. Eldar. This work was supported by the Swiss National Science Foundation (SNSF) by Grant 200020-101821.

The authors are with the Biomedical Imaging Group (BIG), Ecole Polytechnique Fédérale De Lausanne (EPFL), CH-1015 Lausanne, Switzerland (e-mail: sathish.ramani@epfl.ch; dimitri.vandeville@epfl.ch; thierry.blu@epfl.ch; michael.unser@epfl.ch).

Digital Object Identifier 10.1109/TSP.2007.908997

${ }^{1}$ Throughout the paper, we are using a normalized sampling step $T=1$ to simplify the notation. multidimensional signals [10]-[14] and for nonideal, bandlimited measurements [15], [16]. In a more general framework, the problem has been generalized to sampling in arbitrary shift-invariant spaces [17]-[20], Hilbert [21]-[24] and multiresolution subspaces [25]-[28]. Since real-world signals are rarely bandlimited, the recommended procedure is to apply a low-pass filter prior to sampling to avoid aliasing. In practice, this prefilter corresponds to the impulse response of the acquisition device and is generally nonideal. Moreover, the measured samples are often corrupted by noise, an aspect that is not addressed in traditional sampling theory.

Many methods have been proposed to partially compensate for these nonideal conditions by means of digital filtering techniques (inverse filtering or deconvolution) [29]-[32]. In a recent paper, Eldar et al. present an extended formulation for the "optimal" reconstruction of a 1-D signal $f(x)$ from noisy measurements in some general "shift invariant" space $V_{\varphi}=\operatorname{span}\{\varphi(x-k)\}_{k \in \mathbb{Z}}$ where $\varphi$ is an arbitrary generating function [33]. Given some a priori choice of reconstruction space, the optimal approximation is then obtained by suitable digital processing of the nonideal samples. As in the classical case, this corresponds to a special kind of (regularized-inverse, Wiener) filtering, except that the determination of the digital correction filter is slightly more involved because of its explicit dependence on $\varphi$ [33]. The bottom line is that the user is free to select the reconstruction space he wishes, provided of course that he optimizes the digital correction filter accordingly. Having this added flexibility is desirable, but it also raises the important issue of the selection of the "best" reconstruction space for a particular sampling setup and/or class of input signals. This is precisely the question that we will address here while also extending some of the previous formulations to the multidimensional setting. Table I summarizes the characteristics of some well-known methods in literature in comparison to what is developed in this paper.

Is it justifiable or not in practice to depart from the traditional bandlimited formulation? Our answer here is qualified and depends both on the sampling setup and the type of a priori information available on the signal. In contrast with previous works where the solution subspace is fixed a priori, we want to infer the "optimal" reconstruction space together with the reconstruction algorithm in a deductive fashion through a global mathematical optimization process. Specifically, we formulate the problem as an interpolation task wherein the continuousspace solution is obtained by the minimization of a data fidelity term subject to a continuous-space regularization constraint (Tikhonov-like functional). The data fidelity term (which is possibly nonquadratic) ensures that the continuous-space solution is consistent. For the regularization, we choose a convexincreasing function of the $L_{2}$ norm of a generalized derivative 
TABLE I

COMPARISON OF CHARACTERISTICS OF VARIOUS METHODS

\begin{tabular}{|l|c|c|c|c|}
\hline Method & Acquisition device, $h(\mathbf{x})$ & Noise & Reconstruction Space, $\varphi(\mathbf{x})$ & Dimension \\
\hline Shannon [1] & Ideal, $h(\mathbf{x})=\delta(\mathbf{x})$ & No & Bandlimited & 1-D \\
\hline Peterson et al., [10] & Ideal, $h(\mathbf{x})=\delta(\mathbf{x})$ & No & Bandlimited & Multi-D \\
\hline Papoulis [15] & Non-ideal & No & Bandlimited & 1-D \\
\hline Unser et al., [18] & Non-ideal & No & Shift-invariant, Splines & 1-D \\
\hline Eldar et al., [33] & Non-ideal & Yes & Shift-invariant & 1-D \\
\hline This work & Non-ideal & Yes & "Matched" & Multi-D \\
\hline
\end{tabular}

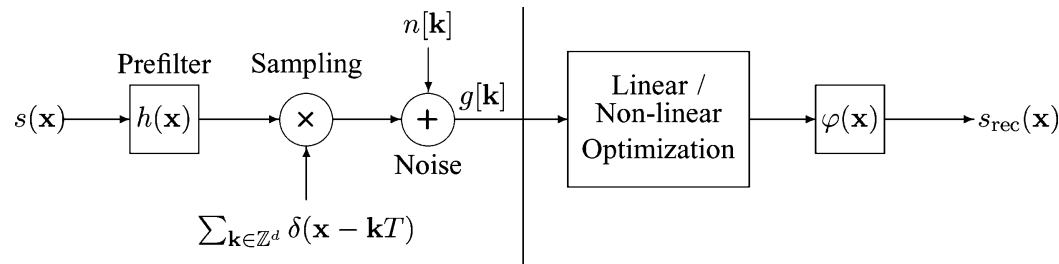

Sampling Setup

Reconstruction

Fig. 1. Block diagram of the generalized sampling problem. The acquisition device specifies the prefilter $h(\mathbf{x})$ which acts in the continuous domain. The choice of the generator $\varphi(\mathbf{x})$ specifies the reconstruction formula. The optimal coefficients $c[\mathbf{k}]$ are determined from the noisy samples $g[\mathbf{k}]$ as the result of a numerical linear/nonlinear optimization process.

of the reconstruction (Tikhonov regularization). This gives rise to a general setting which is mathematically tractable and also leads to the specification of an "optimal" reconstruction space. We will show that the optimal space is shift-invariant in nature and that the continuous-space global minimum solution has an exact analytical form. For a nonquadratic data term, the solution can be computed by nonlinear optimization of the coefficients of its shift-invariant representation. For the quadratic case, we will show that the optimal solution can be obtained by a simple one-step hybrid-linear filtering (discrete in-continuous out) of the measurements. This leads to the reconstruction setup illustrated in Fig. 1.

An important point that is put forth in this work is that the optimal reconstruction space is tied to the choice of regularization operator $\mathrm{L}$ via an optimality condition that will be made explicit. Also, we will argue that the optimal reconstruction space is generally nonbandlimited, unless the impulse response of the acquisition device is itself bandlimited, which is rarely the case in practice. In fact, we will see that the proposed reconstruction framework is closer to spline theory than it is to the traditional view of sampling/deconvolution. The key difference here is that the basis functions are problem-dependent. While this may sound like a complication, the good news for practitioners is that the corresponding computational approach (numerical optimization) is not too different from what they used to be, once the problem has been discretized in the optimal basis. A conceptual advantage is that there are no numerical approximations involved (e.g., finite differences to estimate derivatives, etc.): all calculations are exact and performed analytically in the continuous domain (similar to what is done in the context of the wavelet transform).

We will show that our formulation is general enough to recover most of the smoothing splines estimators that have been proposed in [34]-[37]. We will also present statistical arguments that suggest the same type of estimators and point towards the existence of optimal shift-invariant reconstruction spaces for certain classes of stochastic processes. In fact, we will uncover a functional equivalence between the variational and Wiener solutions to the generalized sampling problem, which in turn helps us to select the most appropriate regularization functional.

The paper is organized as follows. We present our notations and some preliminaries about shift-invariant spaces in Section II. The generalized sampling problem is investigated in Section III. We first give a precise statement of the problem and discuss the mathematical hypotheses that are essential to the analysis. We then derive the general continuous-space solution to our variational reconstruction problem and prove that it is included in some optimal shift-invariant space. An important aspect of the formulation is the characterization of the optimal generator of the solution space which is tightly linked to the regularization operator. Section IV is dedicated to the analysis of the stability of the reconstruction; the end result is a set of relatively mild constraints on the frequency response of the regularization operator that simultaneously ensure that: 1) there is a stable representation (Riesz basis) of the solution space (SR condition), and 2) the reconstruction problem is well posed (CS Condition). We illustrate our results with some concrete examples in Section V. In particular, we consider explicit classes of separable and isotropic regularization operators and characterize the corresponding reconstruction spaces. This also leads to the definition of a new brand of multidimensional "Matérn" splines, which extend Rabut's polyharmonic family. Finally, in Section VI, we revisit our sampling problem from an estimation theoretic point of view and derive the corresponding minimax and MMSE solutions. This allows us to draw an interesting link with the variational formulation leading to some equivalences of solutions for the deterministic and stochastic cases. 


\section{PRELIMINARIES}

\section{A. Notations}

Throughout this paper, we consider real-valued $d$-dimensional signals. Continuous-space signals are denoted with parentheses, e.g., $f(\mathbf{x}): \mathbb{R}^{d} \rightarrow \mathbb{R}, \mathbf{x}=\left(x_{1}, \cdots, x_{d}\right) \in \mathbb{R}^{d}$, and discrete-space signals with brackets, e.g., $c[\mathbf{k}], \mathbf{k} \in \mathbb{Z}^{d}$. We write flipped functions or sequences with a bar; for example, $\bar{f}(\mathbf{x})=f(-\mathbf{x})$ and $\bar{h}[\mathbf{k}]=h[-\mathbf{k}]$.

The continuous-space Fourier transform of a signal $f(\mathbf{x}) \in$ $L_{1}\left(\mathbb{R}^{d}\right) \bigcap L_{2}\left(\mathbb{R}^{d}\right)$ is

$$
\mathcal{F}\{f\}=\hat{f}(\boldsymbol{\omega})=\int_{\mathbb{R}^{d}} f(\mathbf{x}) e^{-j \boldsymbol{\omega}^{\mathrm{T}} \mathbf{x}} d \mathbf{x}
$$

and the discrete-space Fourier transform of a sequence $c[\mathbf{k}] \in$ $\ell_{1}\left(\mathbb{Z}^{d}\right)$ is defined as

$$
C\left(e^{j \boldsymbol{\omega}}\right)=\sum_{\mathbf{k} \in \mathbb{Z}^{d}} c[\mathbf{k}] e^{-j \boldsymbol{\omega}^{\mathrm{T}} \mathbf{k}}
$$

We denote the $L_{2}$-inner product between $f(\mathbf{x})$ and $g(\mathbf{x})$ by

$$
\langle f, g\rangle_{L_{2}}=\int_{\mathbb{R}^{d}} f^{*}(\mathbf{x}) g(\mathbf{x}) d \mathbf{x}
$$

and the $\ell_{2}$-inner product between two complex sequences $c[\mathbf{k}]$ and $b[\mathbf{k}]$ by

$$
\langle c, b\rangle_{\ell_{2}}=\sum_{\mathbf{k} \in \mathbb{Z}^{d}} c^{*}[\mathbf{k}] b[\mathbf{k}]
$$

Continuous-space convolution of $f(\mathbf{x})$ and $g(\mathbf{x})$ is denoted by $(f \star g)(\mathbf{x})$ using the $\star$ symbol, while its discrete counterpart is denoted by $(c * b)[\mathrm{k}]$ using the $*$ symbol, respectively.

We will frequently rely on Parseval's identity that states that for $f(\mathbf{x}), g(\mathbf{x}) \in L_{2}\left(\mathbb{R}^{d}\right)$

$$
\langle f, g\rangle_{L_{2}}=\frac{1}{(2 \pi)^{d}} \int_{\mathbb{R}^{d}} \hat{f}^{*}(\boldsymbol{\omega}) \hat{g}(\boldsymbol{\omega}) d \boldsymbol{\omega}
$$

and for two complex sequences $c[\mathbf{k}], b[\mathbf{k}] \in \ell_{2}\left(\mathbb{Z}^{d}\right)$,

$$
\langle c, b\rangle_{\ell_{2}}=\frac{1}{(2 \pi)^{d}} \int_{[0} C_{2 \pi)^{d}}^{*}\left(e^{j \boldsymbol{\omega}}\right) B\left(e^{j \boldsymbol{\omega}}\right) d \boldsymbol{\omega}
$$

where $[02 \pi)^{d}=\left\{\boldsymbol{\omega}=\left(\omega_{1}, \omega_{2}, \ldots, \omega_{d}\right) \mid \omega_{i} \in[02 \pi), i=\right.$ $1,2, \ldots, d\}$.

We will also use the well known inclusion-property of the $\ell_{p}$ spaces:

$$
\text { for } 1 \leq p_{0}<p_{1} \leq \infty, \ell_{p_{0}}\left(\mathbb{Z}^{d}\right) \subset \ell_{p_{1}}\left(\mathbb{Z}^{d}\right) \text {. }
$$

Using Young's inequality [38], we have for any $f \in L_{1}\left(\mathbb{R}^{d}\right)$ and $g \in L_{1}\left(\mathbb{R}^{d}\right) \bigcap L_{2}\left(\mathbb{R}^{d}\right),(f \star g) \in L_{1}\left(\mathbb{R}^{d}\right) \bigcap L_{2}\left(\mathbb{R}^{d}\right)$. The same holds true for sequences, with $L_{p}(\mathbb{R})$ being replaced by $\ell_{p}\left(\mathbb{Z}^{d}\right)$.

\section{B. Shift-Invariant Spaces}

We briefly review some general results on integer-shift-invariant (or spline-like) spaces and the conditions that must be satisfied by the generating function $\varphi(\mathbf{x})$. The reconstruction space generated by $\varphi(\mathbf{x})$ is defined as

$$
V_{\varphi}=\left\{s(\mathbf{x})=\sum_{\mathbf{k} \in \mathbb{Z}^{d}} c[\mathbf{k}] \varphi(\mathbf{x}-\mathbf{k}): c \in \ell_{2}\left(\mathbb{Z}^{d}\right)\right\} .
$$

For the expansion in (8) to be well defined, $\varphi(\mathbf{x})$ must satisfy some stability conditions.

Definition 1: A function $\varphi(\mathbf{x}): \mathbb{R}^{d} \rightarrow \mathbb{R}$ is a stable generator of $V_{\varphi}$ if and only if it satisfies the two "stable representation" SR conditions:

$$
\mathbf{S R}:\left\{\begin{array}{l}
A_{\varphi, 2}=\inf _{\boldsymbol{\omega} \in[02 \pi)^{d}} \sqrt{\sum_{\mathbf{k} \in \mathbb{Z}^{d}}|\hat{\varphi}(\boldsymbol{\omega}+2 \pi \mathbf{k})|^{2}}>0 \\
\sup _{\mathbf{x} \in[01)^{d}} \sum_{\mathbf{k} \in \mathbb{Z}^{d}}|\varphi(\mathbf{x}+\mathbf{k})|<\infty .
\end{array}\right.
$$

In particular, (9) ensures that the set of functions $\{\varphi(\mathbf{x}-\mathbf{k})\}$ forms an $L_{p}$-stable Riesz basis for all $1 \leq p \leq \infty$ [19], that is, $\forall c \in \ell_{p}$

$$
A_{\varphi, p}\|c\|_{\ell_{p}} \leq\left\|\sum_{\mathbf{k} \in \mathbb{Z}^{d}} c[\mathbf{k}] \varphi(\mathbf{x}-\mathbf{k})\right\|_{L_{p}} \leq B_{\varphi, p}\|c\|_{\ell_{p}}
$$

where $A_{\varphi, p}>0$ and $B_{\varphi, p}<+\infty$ are appropriate constants. The quantity $A_{\varphi, 2}$ in (9) [or in (10) with $p=2$ ] is called the lower Riesz bound and is complementary to the upper Riesz bound $B_{\varphi, 2}$, which is given by

$$
B_{\varphi, 2}=\sup _{\boldsymbol{\omega} \in[02 \pi)^{d}} \sqrt{\sum_{\mathbf{k} \in \mathbb{Z}^{d}}|\hat{\varphi}(\boldsymbol{\omega}+2 \pi \mathbf{k})|^{2}} .
$$

The basis is orthonormal iff $A_{\varphi, 2}=B_{\varphi, 2}=1$. The norm equivalence (10) implies that $V_{\varphi}$ is a closed subspace of $L_{p}\left(\mathbb{R}^{d}\right)$ for all $1 \leq p \leq \infty$. The key point is that any function $s(\mathbf{x}) \in V_{\varphi}$ has a stable and unambiguous representation in terms of its coefficients $c[\mathbf{k}], \mathbf{k} \in \mathbb{Z}^{d}$.

It is important to note that the generator $\varphi$ of $V_{\varphi}$ is by no means unique, as expressed by the following proposition (cf. [32]).

Proposition 1: Let $\varphi$ be a stable generator of $V_{\varphi}$. Then, any function of the form $\varphi_{\mathrm{eq}}(\mathbf{x})=\sum_{\mathbf{k} \in \mathbb{Z}^{d}} q[\mathbf{k}] \varphi(\mathbf{x}-\mathbf{k})$, generates an equivalent Riesz basis of $V_{\varphi}$, if and only if $0<A \leq$ $\left|Q\left(e^{j \omega}\right)\right| \leq B<\infty$, a.e., where $Q\left(e^{j \omega}\right)$ is the Fourier transform of $q[\mathbf{k}]$.

We will also take advantage of the following Young-type inequality which asserts that the second $\mathbf{S R}$ condition is preserved through convolution.

Proposition 2: Let $\varphi_{1}(\mathbf{x})$ satisfy the second $\mathbf{S R}$ condition and let $\varphi_{2}(\mathbf{x})=\left(f \star \varphi_{1}\right)(\mathbf{x})$ for some $f(\mathbf{x}) \in L_{1}\left(\mathbb{R}^{d}\right)$. Then we have

$$
\sup _{x \in[01)^{d}} \sum_{\mathbf{k} \in \mathbb{Z}^{d}}\left|\varphi_{2}(\mathbf{x}+\mathbf{k})\right|<\infty \text {. }
$$


Proof: Consider the series

$$
\begin{aligned}
\sum_{\mathbf{k} \in \mathbb{Z}^{d}}\left|\varphi_{2}(\mathbf{x}+\mathbf{k})\right| & =\sum_{\mathbf{k} \in \mathbb{Z}^{d}}\left|\int_{\mathbb{R}^{d}} f\left(\mathbf{x}^{\prime}\right) \varphi_{1}\left(\mathbf{x}+\mathbf{k}-\mathbf{x}^{\prime}\right) d \mathbf{x}^{\prime}\right| \\
& \leq \int_{\mathbb{R}^{d}}\left|f\left(\mathbf{x}^{\prime}\right)\right|\left(\sum_{\mathbf{k} \in \mathbb{Z}^{d}}\left|\varphi_{1}\left(\mathbf{x}+\mathbf{k}-\mathbf{x}^{\prime}\right)\right|\right) d \mathbf{x}^{\prime} \\
& \leq\|f\|_{L_{1}}\left(\sup _{\mathbf{x}^{\prime} \in[01)^{d}} \sum_{\mathbf{k} \in \mathbb{Z}^{d}}\left|\varphi_{1}\left(\mathbf{x}^{\prime}+\mathbf{k}\right)\right|\right) \\
& <\infty
\end{aligned}
$$

where this inequality holds $\forall \mathbf{x} \in\left[\begin{array}{ll}01 & 1\end{array}{ }^{d}\right.$, including the value $\mathbf{x}_{0}$ for which the left-hand side (LHS) achieves its maximum.

Remark 1: It should be noted that $\left(f \star \varphi_{1}\right)(\mathbf{k}) \in \ell_{1}\left(\mathbb{Z}^{d}\right)$, which is obvious when we set $\mathbf{x}=\mathbf{0}$ in (12).

\section{SAMPLING PROBLEM AND REGULARIZATION THEORY}

\section{A. Problem Specification}

The problem we consider is the recovery of a continuousspace real-valued signal $s(\mathbf{x})$ given some equally spaced, noisy measurements $g[\mathbf{k}]$. The deterministic signal $s(\mathbf{x})$ is convolved with a prefilter $h(\mathbf{x})$ prior to sampling and the generalized samples are corrupted by additive noise. Specifically, the measurement model associated with Fig. 1 is

$$
g[\mathbf{k}]=(h \star s)(\mathbf{k})+n[\mathbf{k}]=y[\mathbf{k}]+n[\mathbf{k}]
$$

where $g[\mathbf{k}]$ are the measurements, $y[\mathbf{k}]=(h \star s)(\mathbf{k})$ are the generalized samples (samples of the prefiltered signal) and $n[\mathbf{k}]$ is a discrete additive, zero-mean noise component.

Throughout this paper, we assume that the analog prefilter $h(\mathbf{x})$, which represents the point spread function (PSF) of the nonideal acquisition device, is of either forms as follows:

(i) PSF defined over a continuum: $h(\mathbf{x}) \in L_{1}\left(\mathbb{R}^{d}\right)$;

(ii) Sampled PSF (or discrete filter): $h(\mathbf{x})=$ $\sum_{\mathbf{k} \in \mathbb{Z}^{d}} h_{d}[\mathbf{k}] \delta(\mathbf{x}-\mathbf{k})$, where $h_{d}[\mathbf{k}] \in \ell_{1}\left(\mathbb{Z}^{d}\right)$.

A necessary condition for the sampling problem to be well defined is that the prefilter be BIBO stable, as implied by (i) or (ii). The PSF in (ii) is not rigorously in $L_{1}\left(\mathbb{R}^{d}\right)$; however, it can be shown that it satisfies all corresponding Young-type inequalities. Note that (ii) also includes the identity filter $h(\mathbf{x})=\delta(\mathbf{x})$ (ideal sampling) as a special case.

We adopt a variational approach and formulate the reconstruction problem as a minimization problem. The solution is obtained by minimizing an error criterion denoted by $\mathcal{J}(g, u)$ which depends on the input measurements $g[\mathbf{k}]$ and the continuous-space reconstruction $u(\mathbf{x})$. The specification of the costfunctional $\mathcal{J}(g, u)$ is based on the following two key points: (a) the reconstruction should be sufficiently constrained (e.g., smooth or slowly varying) to make up for the fact that we are missing information in between pixels and to countermand the effect of noise, and (b) the generalized samples corresponding to the reconstruction should be close to the given measurements to ensure some level of consistency. Specifically, among all continuously defined functions $u(\mathbf{x}) \in L_{2}\left(\mathbb{R}^{d}\right)$, we are seeking the optimal signal reconstruction

$$
s_{\text {rec }}(\mathbf{x})=\arg \min _{u \in L_{2}\left(\mathbb{R}^{d}\right)} \mathcal{J}(g, u)
$$

where the cost functional $\mathcal{J}(g, u)$ is given by

$$
\mathcal{J}(g, u)=\underbrace{\sum_{\mathbf{k} \in \mathbb{Z}^{d}}|g[\mathbf{k}]-(h \star u)(\mathbf{k})|^{p}}_{\text {Data Term }}+\lambda \underbrace{\Phi\left(\|\mathrm{L}\{u\}\|_{L_{2}}^{2}\right)}_{\text {Regularization }} .
$$

$\mathrm{L}$ is a suitable shift-invariant differential (or regularization) operator, $\lambda$ a positive real number, and $\Phi(\cdot)$ is a convex increasing function. The $L_{2}$-norm in the regularization term is a measure of the "roughness" of the reconstruction. Minimization of $\mathcal{J}(g, u)$ therefore ensures sufficient smoothness in the reconstruction while the data term constrains the generalized samples of the reconstruction to be "close" to the measurements in the $\ell_{p}$-sense. The parameter $\lambda \geq 0$ controls the amount of regularization imposed on the reconstruction.

The above cost functional reduces to the well-known Tikhonov criterion when $p=2$ and $\Phi(x)=x$. Moreover, we will show in Section VI that the solution corresponding to the Tikhonov criterion is functionally equivalent to the ones obtained for the minimax estimation and the stochastic (or Wiener) formulation of the generalized sampling problem.

At this point, it is important to note that $\mathcal{J}(g, u)$ is a hybrid criterion that has a discrete part - the data term — and an analog one-the regularization functional-that imposes smoothness constraints on the continuous-domain solution. It is this latter term together with the extent of the search space ${ }^{2}$ that differentiates our problem from a more traditional deconvolution task which is usually entirely formulated in the discrete domain. Here, we are attempting to solve the deconvolution and interpolation problems simultaneously and hoping that the criterion will dictate an "optimal" discretization procedure.

We believe that the present cost-functional is the most general one that can be solved analytically in the continuous-space domain. It allows for a nonquadratic data fidelity term in the spirit of Fu et al. [39] and Nikolova [40], but it excludes some popular nonquadratic regularization such as TV [41], [42], which are not mathematically tractable in the present continuous-space framework. We must admit that this restriction constitutes a limitation of our formulation, but it is also clear that the generalized sampling problem is more difficult than the classical deconvolution problem: we are not only trying to get the optimal solution at the sample locations, but also in-between pixels, which adds another level of ill-posedness.

As we shall see, it is the presence of the $L_{2}$ norm of $\mathrm{L}\{\cdot\}$ in the regularization term that makes the derivation of the continuous-space solution feasible. Indeed, we will show that the continuous-space solution is well defined and that it belongs to

\footnotetext{
${ }^{2}$ We are optimizing the criterion over $L_{2}\left(\mathbb{R}^{d}\right)$, which is considerably larger than the subspace of bandlimited functions. To make an analogy, $L_{2}\left(\mathbb{R}^{d}\right)$ is to $V_{\text {sinc }}$-or, equivalently, $\ell_{2}\left(\mathbb{Z}^{d}\right)$-what the real numbers are to the integers.
} 
an "optimal" subspace, $V_{\varphi_{\text {opt }}}$, that is shift-invariant and independent upon the input signal. Moreover, for $p=2$, there is a direct solution that can be computed elegantly by simple digital filtering of the discrete input measurements. This is illustrated in Fig. 1 in the reconstruction setup where the measurements $g[\mathbf{k}]$ are first compensated for their nonidealness using linear/nonlinear optimization techniques (that can be implemented efficiently via digital filtering); the reconstruction then is performed in the shift-invariant space generated by $\varphi=\varphi_{\mathrm{opt}}$.

\section{B. Consistent Sampling in $V_{\varphi}$}

In the noise-free case, it is reasonable to decrease the weight of the regularization $(\lambda \rightarrow 0)$ and to seek a signal reconstruction that is consistent with the measurements. This corresponds to the case where the samples of the continuous-space function are equal to the measurements (so that the data term is zero). This is the approach to the generalized sampling problem that was developed initially in [32] for a signal reconstruction in some predefined shift-invariant space $V_{\varphi}$. We will review this solution here, keeping in mind that it is not necessarily optimal because of the restriction on the search spaces (i.e., $V_{\varphi}$ instead of $L_{2}\left(\mathbb{R}^{d}\right)$ ). Under suitable conditions (cf. [32, Theorem 1]), the consistent signal reconstruction in $V_{\varphi}$ is unique and is given by $s_{\text {con }}(\mathbf{x})=\sum_{\mathbf{k} \in \mathbb{Z}^{d}}\left(g * r_{0}\right)[\mathbf{k}] \varphi(\mathbf{x}-\mathbf{k})$, where $r_{0}$ is the digital restoration filter whose frequency response is [32]

$$
R_{0}\left(e^{j \boldsymbol{\omega}}\right)=\frac{1}{\sum_{\mathbf{k} \in \mathbb{Z}^{d}} \hat{h}(\boldsymbol{\omega}+2 \pi \mathbf{k}) \hat{\varphi}(\boldsymbol{\omega}+2 \pi \mathbf{k})} .
$$

Note that this filter corresponds to the convolution inverse of the sequence $(h \star \varphi)(\mathbf{k})$. We check for the consistency of $\left(h \star_{\text {con }}\right)(\mathbf{x})$ by sampling it at the integers: $\left.\left(h \star s_{\text {con }}\right)(\mathbf{x})\right|_{\mathbf{x}=\mathbf{n} \in \mathbb{Z}^{d}}=\sum_{\mathbf{k} \in \mathbb{Z}^{d}}\left(g * r_{0}\right)[\mathbf{k}](h \star \varphi)(\mathbf{n}-\mathbf{k})=g[\mathbf{n}]$. This holds true because $r_{0} *(h \star \varphi)(\mathbf{n})=\delta[\mathbf{n}]$ and the interchange of the sum and integral is justified using Lebesgue's dominated convergence theorem in combination with Schwarz's inequality. The condition for the existence (and unicity) of the consistent sampling (CS) solution is that the denominator of (16) is nonvanishing:

$$
\mathbf{C S}:\left|\sum_{\mathbf{k} \in \mathbb{Z}^{d}} \hat{h}(\boldsymbol{\omega}+2 \pi \mathbf{k}) \hat{\varphi}(\boldsymbol{\omega}+2 \pi \mathbf{k})\right|>0 \quad \forall \boldsymbol{\omega} \in[02 \pi)^{d}
$$

which imposes a joint constraint on $\varphi(\mathbf{x})$ and $h(\mathbf{x})$. This guarantees the BIBO stability of the reconstruction filter $r_{0}$. Indeed, the $L_{p}$-stability of $\varphi$ (SR condition) implies that $(h \star \varphi)(\mathbf{k}) \in$ $\ell_{1}\left(\mathbb{Z}^{d}\right)$ [see Remark 1]. The results then follow from Wiener's lemma [43], which ensures that the inverse filter $r_{0}[\mathbf{k}] \in \ell_{1}\left(\mathbb{Z}^{d}\right)$. The argument also holds in the case where $h$ is a sampled PSF.

\section{Space of Admissible Solutions}

We now go back to our initial minimization problem (14) with $\lambda>0$. While searching for a global solution in $L_{2}\left(\mathbb{R}^{d}\right)$, we must exclude all potential candidates for which the cost is infinite. We therefore say that the function $u(\mathbf{x}) \in L_{2}\left(\mathbb{R}^{d}\right)$ is admissible with respect to the criterion (14) if and only if $\mathcal{J}(g, u)$ is finite. In particular, this implies that

$$
\|\mathrm{L}\{u\}\|_{L_{2}}^{2}=\int_{\mathbf{x} \in \mathbb{R}^{d}}|\mathrm{~L}\{u(\mathbf{x})\}|^{2} d \mathbf{x} \leq \mu_{0}^{2}<+\infty .
$$

This together with the fact that we are looking for a solution with finite $L_{2}$ norm gets translated into $u(\mathbf{x}) \in W_{L}^{2}$, where $W_{L}^{2}=\left\{f(\mathbf{x}): \int_{\mathbb{R}^{d}}|\hat{f}(\boldsymbol{\omega})|^{2}\left(1+|\hat{\mathrm{L}}(\boldsymbol{\omega})|^{2}\right) d \boldsymbol{\omega}<+\infty\right\}$ is the generalized Sobolev space associated with the operator L. Thus, the problem can be restated as

$$
s_{\text {rec }}(\mathbf{x})=\arg \min _{u \in W_{2}^{\mathrm{L}}} \mathcal{J}(g, u)
$$

Additionally, for the data term in $\mathcal{J}(g, u)$ to be finite (in the $p=2$ case), the samples of the solution $s_{\text {rec }} \in W_{L}^{2}$ should be well-defined in the $\ell_{2}$-sense (we examine the cases where $p \neq 2$ when we actually present the solution to the minimization problem). This is ensured provided that $\mathrm{L}$ acts as a differential operator and enforces sufficient smoothness on the solution. In multiple dimensions, a classical choice for $\mathrm{L}$ is the $(\gamma / 2)$-iterated multidimensional Laplacian operator $(\gamma>0)$, whose transfer function is $\hat{\mathrm{L}}(\boldsymbol{\omega})=\|\boldsymbol{\omega}\|^{\gamma}$ [35], [44], [45]. This leads to the Sobolev space $W_{2}^{\gamma}$ of order $\gamma$ that contains finite energy functions whose derivatives up to order $\gamma$ have finite $L_{2}$-norm [34], [45]. Among other properties of $W_{2}^{\gamma}$, the one that is of interest to us is: for real $\gamma>(d / 2), f(\mathbf{x}) \in W_{2}^{\gamma} \Longrightarrow$ $\left.f(\mathbf{k})\right|_{\mathbf{k} \in \mathbb{Z}^{d}} \in \ell_{2}\left(\mathbb{Z}^{d}\right)$ (see [46, Appendix C] for the proof when $d=1)$.

In this paper, we propose to extend this result to a larger class of multidimensional differential operator L. To that end, we impose the following admissibility condition on L which guarantees the minimum required degree of smoothness.

Definition 2: L is an admissible multidimensional differential operator if and only if

$$
\sum_{\mathbf{k} \in \mathbb{Z}^{d}} \frac{1}{1+|\hat{\mathrm{L}}(\boldsymbol{\omega}+2 \pi \mathbf{k})|^{2}} \leq C_{0}<+\infty
$$

This condition implicitly controls the minimal "growth" rate of $\hat{\mathrm{L}}(\boldsymbol{\omega})$, because the above series converges only when $\hat{\mathrm{L}}(\boldsymbol{\omega})$ grows faster than $\|\boldsymbol{\omega}\|^{(d / 2)}$ which is the limit case. If the generalized differential operator $\mathrm{L}$ is admissible, then the following theorem ensures that the associated generalized Sobolev space $W_{L}^{2}$ has the properties that we demand.

Theorem 1: Let $W_{L}^{2}$ be the generalized Sobolev space associated with the admissible regularization operator L. Then $f(\mathbf{x}) \in$ $W_{L}^{2} \Longrightarrow\{f(\mathbf{k})\}_{\mathbf{k} \in \mathbb{Z}^{d}} \in \ell_{2}\left(\mathbb{Z}^{d}\right)$, and the Poisson summation formula holds: $\sum_{\mathbf{k} \in \mathbb{Z}^{d}} f(\mathbf{k}) e^{-j \boldsymbol{\omega}^{\mathrm{T}} \mathbf{k}}=\sum_{\mathbf{k} \in \mathbb{Z}^{d}} \hat{f}(\boldsymbol{\omega}+2 \pi \mathbf{k})$ a.e.

The proof is given in Appendix A.

For the present context, we extend the scope of the above theorem to functions of type $(h \star f)(\mathbf{x})$ using the following proposition.

Corollary 1: If $f(\mathbf{x}) \in W_{L}^{2}$ and $h(\mathbf{x}) \in L_{1}\left(\mathbb{R}^{d}\right)$, then $(h \star$ $f)(\mathbf{x}) \in W_{L}^{2}$ and $(h \star f)(\mathbf{k}) \in \ell_{2}\left(\mathbb{Z}^{d}\right)$. 
Proof: Since $h(\mathbf{x}) \in L_{1}\left(\mathbb{R}^{d}\right),|\hat{h}(\boldsymbol{\omega})| \leq\|h\|_{L_{1}}<+\infty$, and the result is obvious from the definition of $W_{L}^{2}$ and the application of Theorem 1. The same holds for the sampled PSF case too.

It is also clear that the search for a suboptimal solution in some subspace $V_{\varphi}$ only makes sense if $V_{\varphi} \subset W_{L}^{2}$. This is equivalent to requiring that the upper Riesz bound $B_{\mathrm{L} \varphi, 2}$, as defined by (11), be finite.

\section{Solution to the Variational Problem}

To minimize the cost functional $\mathcal{J}(g, u)$, we first observe that the data term depends on the generalized samples of the solution exclusively, which suggests a two-stage optimization strategy. We first deal with the regularization part of the problem. Let us denote by $u_{0}(\mathbf{x})$ the optimal solution to our problem. The main part of the argument will be the construction of a function $s_{\text {con }}(\mathbf{x})$ which belongs to some "optimal" shift-invariant space $V_{\varphi_{\text {opt }}}$, that is consistent with $u_{0}(\mathbf{x})$ (in the sense that $\left.y[\mathbf{k}]=\left(h \star u_{0}\right)(\mathbf{k})=\left(h \star s_{\text {con }}\right)(\mathbf{k})\right)$ and which has the least $\Phi\left(\|\mathrm{L}\{\cdot\}\|_{L_{2}}^{2}\right)$ among all consistent functions in $W_{L}^{2}$ (Section III-D-1). This leads us to conclude that there is a solution of our global optimization problem that belongs to the space $V_{\varphi_{\mathrm{opt}}}$ (Sections III-D-2 and D-3). Once the optimal space is known, we only need to determine the coefficients $c[\mathbf{k}]$ of the shift-invariant representation which yields the discretized version of the problem presented in Section III-D-4. The quadratic case, which can be solved explicitly, is dealt with in Section III-D-5.

1) Consistent, Shift-Invariant Solution: The optimal generating function for our problem will be denoted by $\varphi_{\mathrm{opt}}(\mathbf{x})$. We also introduce a sufficient condition for optimality which will be justified by Theorem 2 .

Definition 3: Let $\varphi_{\mathrm{opt}}(\mathbf{x})$ be a generating function that satisfies the $\mathbf{S R}$ and $\mathbf{C S}$ conditions for a given $h$. Then, $\varphi_{\text {opt }}(\mathbf{x})$ is said to be optimal with respect to the problem (14) if there exists a sequence $q[\mathbf{k}] \in \ell_{1}\left(\mathbb{Z}^{d}\right)$ such that

Optimality Condition OC :

$$
\mathrm{L}^{*} \mathrm{~L}\left\{\varphi_{\mathrm{opt}}(\mathbf{x})\right\}=\sum_{\mathbf{k} \in \mathbb{Z}^{d}}(q * \bar{q})[\mathbf{k}] \bar{h}(\mathbf{x}-\mathbf{k})
$$

where $\bar{q}[\mathbf{k}]=q[-\mathbf{k}]$ and $\mathrm{L}^{*} \mathrm{~L}$ is the self-adjoint operator whose transfer function is $|\hat{\mathrm{L}}(\boldsymbol{\omega})|^{2}$.

We now construct a function $s_{\text {con }}(\mathbf{x}) \in V_{\varphi_{\mathrm{opt}}}$ that is consistent with the optimal solution $u_{0}(\mathbf{x})$ and therefore yields the same data term. This can always be done, as stated here.

Proposition 3: Let $\varphi_{\mathrm{opt}}(\mathbf{x})$ be optimal as in Definition 3. Then, if the samples $y[\mathbf{k}]=\left(h \star u_{0}\right)(\mathbf{k}) \in \ell_{2}\left(\mathbb{Z}^{d}\right)$, there exists a unique consistent function $s_{\text {con }}(\mathbf{x}) \in V_{\varphi_{\mathrm{opt}}}$ such that $(h \star$ $\left.u_{0}\right)(\mathbf{k})=\left(h \star s_{\text {con }}\right)(\mathbf{k}), \forall \mathbf{k} \in \mathbb{Z}^{d}$. It is given by

$$
s_{\mathrm{con}}(\mathbf{x})=\sum_{\mathbf{k} \in \mathbb{Z}^{d}} \underbrace{\left(y * r_{0}\right)[\mathbf{k}]}_{c[\mathbf{k}]} \varphi_{\mathrm{opt}}(\mathbf{x}-\mathbf{k})
$$

where the digital reconstruction filter $r_{0}$ is specified by (16) with $\varphi=\varphi_{\text {opt }}$. Moreover, we have the equivalence $\left(h \star u_{0}\right)(\mathbf{k}) \in$ $\ell_{2}\left(\mathbb{Z}^{d}\right) \Longleftrightarrow s_{\text {con }}(\mathbf{x}) \in W_{L}^{2}$.

Proof: The first part of the proposition is a direct application of the consistent sampling solution presented in
Section III-B. Since $r_{0} \in \ell_{1}\left(\mathbb{Z}^{d}\right)$ (CS Condition) and $y[\mathbf{k}]=\left(h \star u_{0}\right)(\mathbf{k}) \in \ell_{2}\left(\mathbb{Z}^{d}\right)$, we have $c=\left(y * r_{0}\right) \in \ell_{2}\left(\mathbb{Z}^{d}\right)$ by Young's inequality. This implies that $\left\|s_{\text {con }}(\mathbf{x})\right\|_{L_{2}}<\infty$, because $\varphi_{\text {opt }}$ generates a Riesz basis. To further prove that $\left\|\mathrm{L}\left\{s_{\text {con }}\right\}\right\|_{L_{2}}<\infty$, we need to show that $\mathrm{L}\left\{\varphi_{\text {opt }}\right\}$ has an upper Riesz bound: By Proposition 2, we have $\left(h \star \varphi_{\text {opt }}\right)(\mathbf{k}) \in \ell_{1}\left(\mathbb{Z}^{d}\right)$. This together with the fact that $\left|Q\left(e^{j \omega}\right)\right|$ is bounded from above yields

$$
\begin{aligned}
& \sum_{\mathbf{k} \in \mathbb{Z}^{d}}\left|\hat{\mathrm{L}}(\boldsymbol{\omega}+2 \pi \mathbf{k}) \hat{\varphi}_{\mathrm{opt}}(\boldsymbol{\omega}+2 \pi \mathbf{k})\right|^{2} \\
& \quad=\left|Q\left(e^{j \boldsymbol{\omega}}\right)\right|^{2}\left(\sum_{\mathbf{k} \in \mathbb{Z}^{d}} \hat{h}(\boldsymbol{\omega}+2 \pi \mathbf{k}) \hat{\varphi}_{\mathrm{opt}}(\boldsymbol{\omega}+2 \pi \mathbf{k})\right) \\
& \quad<\infty
\end{aligned}
$$

which ensures that $B_{\mathrm{L} \varphi_{\mathrm{opt}}, 2}$ is finite. We have, thus, established the forward implication: $\left(h \star u_{0}\right)(\mathbf{k}) \in \ell_{2}\left(\mathbb{Z}^{d}\right) \Longrightarrow s_{\text {con }}(\mathbf{x}) \in$ $W_{L}^{2}$. The converse is a consequence of Theorem 1 .

An interesting property that will be used later on is that the frequency response $R_{0}\left(e^{j \omega}\right)$ of the corresponding optimal reconstruction filter is always strictly positive. This can be seen by writing (21) in the Fourier domain as

$$
\begin{aligned}
\hat{\varphi}_{\mathrm{opt}}(\boldsymbol{\omega}) & =\hat{h}^{*}(\boldsymbol{\omega}) \frac{\left|Q\left(e^{j \omega}\right)\right|^{2}}{|\hat{\mathrm{L}}(\boldsymbol{\omega})|^{2}} \\
\Longrightarrow \hat{h}(\boldsymbol{\omega}) \hat{\varphi}_{\mathrm{opt}}(\boldsymbol{\omega}) & =|\hat{h}(\boldsymbol{\omega})|^{2} \frac{\left|Q\left(e^{j \boldsymbol{\omega}}\right)\right|^{2}}{|\hat{\mathrm{L}}(\boldsymbol{\omega})|^{2}} \geq 0
\end{aligned}
$$

which implies $\sum_{\mathbf{k} \in \mathbb{Z}^{d}} \hat{h}(\boldsymbol{\omega}+2 \pi \mathbf{k}) \hat{\varphi}_{\text {opt }}(\boldsymbol{\omega}+2 \pi \mathbf{k}) \geq 0, \forall \boldsymbol{\omega} \in$ $[02 \pi)^{d}$. In fact, the latter is a strict inequality because of the CS condition.

2) Global Optimality of the Shift-Invariant Solution: Now that we have constructed the consistent function $s_{\text {con }}(\mathbf{x})$, it only remains to show that it is the one with the least $\Phi\left(\|\mathrm{L}\{\cdot\}\|_{L_{2}}^{2}\right)$ value. To do this, we establish a norm identity that decomposes $\|\mathrm{L}\{u\}\|_{L_{2}}^{2}$ into two orthogonal components.

Theorem 2: Let $V_{\varphi_{\mathrm{opt}}} \subset W_{L}^{2}$ be the function space generated by $\varphi_{\text {opt }}$ as in Definition 3. Then, the following orthogonality property holds: $\forall u(\mathbf{x}) \in W_{L}^{2}$

$$
\|\mathrm{L}\{u\}\|_{L_{2}}^{2}=\left\|\mathrm{L}\left\{u-s_{\text {con }}\right\}\right\|_{L_{2}}^{2}+\left\|\mathrm{L}\left\{s_{\text {con }}\right\}\right\|_{L_{2}}^{2}
$$

where $s_{\text {con }}(\mathbf{x})$ is the unique consistent function in $V_{\varphi_{\text {opt }}}$ such that $\left.\left(h \star s_{\text {con }}\right)(\mathbf{x})\right|_{\mathbf{x}=\mathbf{k}}=(h \star u)(\mathbf{k}), \forall \mathbf{k} \in \mathbb{Z}^{d}$, as specified in Proposition 3.

The proof is given in Appendix B.

Setting $u=s_{\text {con }}$ in (24) minimizes the function norm $\|\mathrm{L}\{\cdot\}\|_{L_{2}}$. Thus, $s_{\text {con }}(\mathbf{x}) \in V_{\varphi_{\text {opt }}}$ minimizes $\|\mathrm{L}\{u\}\|_{L_{2}}^{2}$ among all functions in $W_{L}^{2}$ having consistent samples.

3) General Solution: Putting the pieces together, we get our main theorem, which states that the solution to our optimization problem has a unique representation in the integer-shift-invariant space $V_{\varphi_{\mathrm{opt}}}$.

Theorem 3: Given the measurements $g[\mathbf{k}] \in \ell_{p}\left(\mathbb{Z}^{d}\right)$ and the "optimal" subspace of $W_{L}^{2}, V_{\varphi_{\mathrm{opt}}}=\operatorname{span}\left\{\varphi_{\mathrm{opt}}(\mathbf{x}-\mathbf{k})\right\}_{\mathbf{k} \in \mathbb{Z}^{d}}$ 
with $\varphi_{\text {opt }}$ as in Definition 3, one has the following problem equivalence

$$
\min _{u \in W_{2}^{\mathrm{L}}} \mathcal{J}(g, u)=\min _{s \in V_{\varphi_{\mathrm{opt}}}} \mathcal{J}(g, s)
$$

for any general cost function of the form (15).

This implies that the optimal signal reconstruction can be written as $s_{\mathrm{rec}}(\mathbf{x})=\sum_{\mathbf{k} \in \mathbb{Z}^{d}} c[\mathbf{k}] \varphi_{\mathrm{opt}}(\mathbf{x}-\mathbf{k})$ and the optimization performed over the discrete set of coefficients $c[\mathbf{k}] \in$ $\ell_{2}\left(\mathbb{Z}^{d}\right)$, which narrows down the search considerably.

Proof: We first concentrate on the case $p \geq 2$. Since we are looking for a reconstruction $u(\mathbf{x}) \in W_{L}^{2}$, the generalized samples $(h \star u)(\mathbf{k})$ are in $\ell_{2}\left(\mathbb{Z}^{d}\right) \subset \ell_{p}\left(\mathbb{Z}^{d}\right)$ and the cost functional $\mathcal{J}(g, u)$ is always well defined. The cost (15) is convex over $W_{L}^{2}$ because it is the sum of two (strictly) convex sub-functionals. We are therefore guaranteed that the LHS of the problem in (25) has a global minimum associated with the solution $u_{0}(\mathbf{x})$. The corresponding consistent reconstruction in $V_{\varphi_{\mathrm{opt}}}$ is denoted by $s_{\text {con }, 0}(\mathbf{x})$ and is such that $\left(h \star u_{0}\right)(\mathbf{k})=\left(h \star s_{\text {con }, 0}\right)(\mathbf{k}), \forall \mathbf{k} \in$ $\mathbb{Z}^{d}$. Applying Theorem 2 to the regularization part of the criterion, we have

$$
\begin{aligned}
\mathcal{J}(g, u)= & \sum_{\mathbf{k} \in \mathbb{Z}^{d}}|g[\mathbf{k}]-(h \star u)(\mathbf{k})|^{p} \\
& +\lambda \Phi\left(\left\|\mathrm{L}\left\{s_{\text {con }, 0}\right\}\right\|_{L_{2}}^{2}+\left\|\mathrm{L}\left\{u-s_{\text {con }, 0}\right\}\right\|_{L_{2}}^{2}\right)
\end{aligned}
$$

where the data term and the consistent function $s_{\mathrm{con}, 0} \in V_{\varphi_{\mathrm{opt}}}$ are fixed and uniquely tied to $u$. The optimality of $u_{0}$ implies that $\mathcal{J}\left(g, u_{0}\right) \leq \mathcal{J}\left(g, s_{\text {con }, 0}\right)$. On the other hand, the comparison of (26) for $u=u_{0}$ and $u=s_{\text {con, } 0}$ indicates that $\mathcal{J}\left(g, u_{0}\right) \geq \mathcal{J}\left(g, s_{\text {con }, 0}\right)$ because the data term is the same in both cases and the function $\Phi$ is convex increasing. Thus, the conclusion is that $\mathcal{J}\left(g, u_{0}\right)=\mathcal{J}\left(g, s_{\mathrm{con}, 0}\right)$, which proves our assertion.

For $p<2$, the situation is more restricted because the cost $\mathcal{J}(g, u)$ explodes if the samples $\left(h \star u_{0}\right)(\mathbf{k}) \in \ell_{2}\left(\mathbb{Z}^{d}\right) \backslash \ell_{p}\left(\mathbb{Z}^{d}\right)$. The minimization of $\mathcal{J}(g, u)$ therefore automatically confines the samples $\left(h \star u_{0}\right)(\mathbf{k})$ to lie in $\ell_{p}\left(\mathbb{Z}^{d}\right)$. Since $\ell_{p}\left(\mathbb{Z}^{d}\right) \subset \ell_{2}\left(\mathbb{Z}^{d}\right)$ for $1 \leq p<2$, the shift-invariant solution $s_{\mathrm{con}, 0}(\mathbf{x}) \in V_{\varphi_{\mathrm{opt}}}$ is still valid by Proposition 3 and Theorem 2 .

The above result is conceptually pleasing because the continuous-space optimization problem (19) does not make any $a$ priori assumption on the form of the reconstruction. The shiftinvariant structure of the solution comes out as a result of the mathematical optimization. The generator of the optimal reconstruction space is specified by the optimality condition $\mathbf{O C}$, that is, the operator L determines the generator $\varphi_{\text {opt }}(\mathbf{x})$. This simply means that the reconstruction space should be "matched" to the regularization operator.

Note that the fundamental solution of (21)-i.e., $q[\mathbf{k}]=$ $\delta[\mathbf{k}]$-is $\varphi_{\text {opt }}(\mathbf{x})=(\bar{h} \star \rho)(\mathbf{x})$, where $\rho(\mathbf{x})$ is a Green's function of the self-adjoint operator $\mathrm{L}^{*} \mathrm{~L}$, and this generator is generally not bandlimited.

4) Optimal Discretization of the Problem: Once the reconstruction space $V_{\varphi_{\text {opt }}}$ is specified, we only need to search for the expansion coefficients $c[\mathbf{k}] \in \ell_{2}\left(\mathbb{Z}^{d}\right)$ of the solution. To do this, we write $\left(h \star s_{\text {con, } 0}\right)(\mathbf{k})=(c * \xi)(\mathbf{k})$, where $\xi[\mathbf{k}]=$ $\left(h \star \varphi_{\mathrm{opt}}\right)(\mathbf{k})$. Using (21) for the second term, the cost can be rewritten in terms of the signal coefficients $\{c[\mathbf{k}]\}_{\mathbf{k} \in \mathbb{Z}^{d}}$

$$
\begin{aligned}
\mathcal{J}\left(g, s_{\mathrm{rec}}\right) & =\mathcal{J}(g ; c) \\
& =\|g-(c * \xi)\|_{\ell_{p}}^{p}+\lambda \Phi\left(\langle q * \bar{q} * c, \xi * c\rangle_{\ell_{2}}\right)
\end{aligned}
$$

where we have used the fact that $\left\|\mathrm{L}\left\{s_{\text {rec }}\right\}\right\|_{L_{2}}^{2}=$ $\left\langle\mathrm{L} * \mathrm{~L}\left\{s_{\text {rec }}\right\}, s_{\text {rec }}\right\rangle_{L_{2}}=\langle q * \bar{q} * c, \xi * c\rangle_{\ell_{2}}$. We are, therefore, faced with a nonlinear optimization problem. Even though the problem does generally not have an explicit analytical solution, the good news is that $\mathcal{J}(g ; c)$ is a convex function of the coefficients $c$, which ensures that any local minimum automatically yields a global solution. The minimization can therefore be done by using any standard gradient-based nonlinear optimization technique [47].

5) Solution of the Quadratic/Tikhonov Problem: When $p=$ 2 and $\Phi(x)=x$, the cost functional (27) is quadratic in $c$ (Tikhonov Criterion) and the derivation of the optimal solution can be carried out analytically. This yields an efficient digital filtering reconstruction algorithm.

Corollary 2: When $p=2, \Phi(x)=x$ and $g[\mathbf{k}] \in \ell_{2}\left(\mathbb{Z}^{d}\right)$, the global minimum of the cost functional $\mathcal{J}(g, u)$ is achieved by

$$
s_{\mathrm{rec}}(\mathbf{x})=\sum_{\mathbf{k} \in \mathbb{Z}^{d}}\left(r_{\lambda} * g\right)[\mathbf{k}] \varphi_{\mathrm{opt}}(\mathbf{x}-\mathbf{k})
$$

where the frequency response of the optimal restoration (digital correction) filter $r_{\lambda}[\mathrm{k}]$ is given by

$$
R_{\lambda}\left(e^{j \boldsymbol{\omega}}\right)=\frac{1}{\sum_{\mathbf{k} \in \mathbb{Z}^{d}} \hat{h}(\boldsymbol{\omega}+2 \pi \mathbf{k}) \hat{\varphi}_{\mathrm{opt}}(\boldsymbol{\omega}+2 \pi \mathbf{k})+\lambda\left|Q\left(e^{j \boldsymbol{\omega}}\right)\right|^{2}} .
$$

Proof: Setting $p=2$ and $\Phi(x)=x$ in (27) and equating the partial derivative of $\mathcal{J}(g ; c)$ with respect to $c[\mathbf{k}]$ to zero yields

$$
(\bar{\xi} * \xi * c)[\mathbf{k}]+\lambda(\bar{\xi} * q * \bar{q} * c)[\mathbf{k}]=(\bar{\xi} * g)[\mathbf{k}] .
$$

Rewriting this equality in the Fourier domain, we obtain the desired result

$$
\begin{aligned}
C\left(e^{j \boldsymbol{\omega}}\right) & =\frac{G\left(e^{j \boldsymbol{\omega}}\right)}{\sum_{\mathbf{k} \in \mathbb{Z}^{d}} \hat{\varphi}_{\mathrm{opt}}(\boldsymbol{\omega}+2 \pi \mathbf{k}) \hat{h}(\boldsymbol{\omega}+2 \pi \mathbf{k})+\lambda\left|Q\left(e^{j \boldsymbol{\omega}}\right)\right|^{2}} \\
& =R_{\lambda}\left(e^{j \boldsymbol{\omega}}\right) G\left(e^{j \boldsymbol{\omega}}\right)
\end{aligned}
$$

where $G\left(e^{j \boldsymbol{\omega}}\right)$ is the Fourier transform of the given samples $g[\mathbf{k}]$.

Since $R_{0}\left(e^{j \boldsymbol{\omega}}\right)$ is strictly positive and because $Q\left(e^{j \boldsymbol{\omega}}\right)$ is bounded, the filter $R_{\lambda}\left(e^{j \omega}\right)$ is strictly positive and bounded as well. Therefore, by writing the solution (28) in the Fourier domain, we obtain

$$
\hat{s}_{\text {rec }}(\boldsymbol{\omega})=\hat{\varphi}_{\mathrm{eq}}(\boldsymbol{\omega}) G\left(e^{j \boldsymbol{\omega}}\right)
$$

where $\hat{\varphi}_{\mathrm{eq}}(\boldsymbol{\omega})=R_{\lambda}\left(e^{j \boldsymbol{\omega}}\right) \hat{\varphi}_{\mathrm{opt}}(\boldsymbol{\omega})$ is the equivalent basis function that needs to be applied to the measurements $G\left(e^{j \boldsymbol{\omega}}\right)$ to produce the continuous-space signal reconstruction, as illustrated in Fig. 2. Indeed, we are ensured that $\varphi_{\mathrm{eq}}(\mathbf{x})$ generates a $L_{p}$-stable 


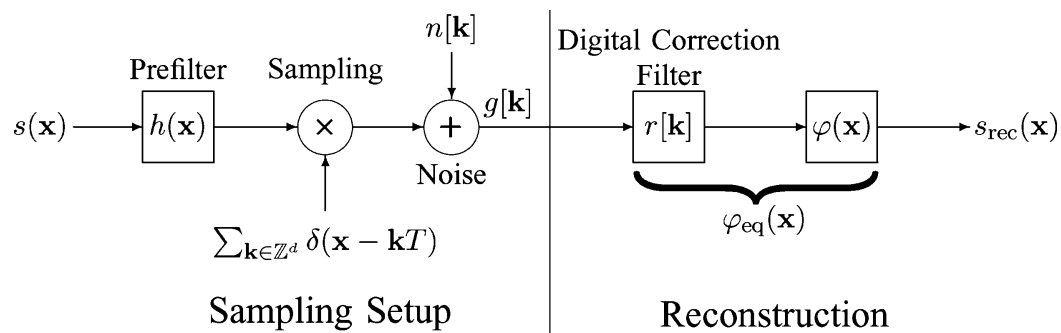

Fig. 2. Reconstruction for the case of $p=2$ : Linear filtering of measurements $g[\mathbf{k}]$.

Riesz basis because of the boundedness of $R_{\lambda}\left(e^{j \omega}\right)$ (see Proposition 1).

\section{StabiLity OF THE RECONSTRUCTION}

In this section, we examine $\varphi_{\text {opt }}$ from a spline-theoretic point of view and provide guidelines for selecting $\mathrm{L}$ so that the problem is well posed. To this end, we rewrite the optimality condition (23) in the Fourier domain as $\hat{\varphi}_{\text {opt }}(\boldsymbol{\omega})=\hat{h}^{*}(\boldsymbol{\omega})|\hat{\beta}(\boldsymbol{\omega})|^{2}$ where

$$
\hat{\beta}(\boldsymbol{\omega})=\frac{Q\left(e^{j \boldsymbol{\omega}}\right)}{\hat{\mathrm{L}}(\boldsymbol{\omega})}
$$

is interpreted as the Fourier transform of the generalized B-spline associated with the operator L [34], [37]. The construction of a B-spline is typically achieved by selecting a filter $Q\left(e^{j \boldsymbol{\omega}}\right)$ that cancels the zeros of $\hat{\mathrm{L}}(\boldsymbol{\omega})$ in order to produce a frequency response $\hat{\beta}(\boldsymbol{\omega})$ that is bounded. In the sequel, we will only consider "spline admissible" operators for which the corresponding generalized B-spline generates an $L_{p}$-stable Riesz basis.

Definition 4: The operator $\mathrm{L}$ is said to be spline-admissible with B-spline $\beta(\mathbf{x})$ iff

$$
\mathrm{L}\{\beta\}=\sum_{\mathbf{k} \in \mathbb{Z}^{d}} q[\mathbf{k}] \delta(\mathbf{x}-\mathbf{k})
$$

where $q[\mathbf{k}] \in \ell_{1}\left(\mathbb{Z}^{d}\right)$, and $\beta(\mathbf{x})$ satisfies the $\mathbf{S R}$ condition.

Note that the above equation is just a restatement of (31) in the signal domain. The nontrivial aspect here is the existence of the lower Riesz bound $A_{\beta, 2}$, which needs to be checked on a case-by-case basis.

The optimal generating function can therefore be regarded a compound generalized B-spline given by

$$
\varphi_{\mathrm{opt}}(\mathbf{x})=(\bar{h} \star \beta \star \bar{\beta})(\mathbf{x}) \text {. }
$$

We will now see that this interpretation of $\varphi_{\text {opt }}$ greatly simplifies our task of making sure that the conditions SR and CS for the well-posedness of our reconstruction problem are met. Interestingly, both conditions are tightly linked when we are working with the optimal basis.

Proposition 4: Let $\varphi_{\text {opt }}$ be the optimal generator. If $h(\mathbf{x}) \in$ $L_{1}\left(\mathbb{R}^{d}\right)$ and $\mathrm{L}$ is spline-admissible, then we have the following equivalence: $\varphi_{\text {opt }}$ satisfies the $\mathbf{S R}$ conditions $\Longleftrightarrow \varphi_{\text {opt }}$ satisfies the CS condition.
Proof: Let us start from the left. Since $\varphi_{\text {opt }}$ satisfies the first $\mathbf{S R}$ condition, we have a strictly positive lower bound for the series

$$
\begin{aligned}
\sum_{\mathbf{k} \in \mathbb{Z}^{d}}\left|\hat{\varphi}_{\mathrm{opt}}(\boldsymbol{\omega}+2 \pi \mathbf{k})\right|^{2} & =\sum_{\mathbf{k} \in \mathbb{Z}^{d}}|\hat{h}(\boldsymbol{\omega}+2 \pi \mathbf{k})|^{2}|\hat{\beta}(\boldsymbol{\omega}+2 \pi \mathbf{k})|^{4} \\
& \geq A_{\varphi_{\mathrm{opt}}, 2}^{2}>0
\end{aligned}
$$

$\forall \boldsymbol{\omega} \in\left[\begin{array}{ll}02 \pi \\ { }^{d}\end{array}\right.$ uous functions of $\boldsymbol{\omega}$, this means that for each $\boldsymbol{\omega}_{0} \in[02 \pi)^{d}$, there is at least one $\mathbf{k} \in \mathbb{Z}^{d}$ such that $\hat{h}\left(\boldsymbol{\omega}_{0}+2 \pi \mathbf{k}\right) \hat{\beta}\left(\boldsymbol{\omega}_{0}+2 \pi \mathbf{k}\right) \neq 0$. Thus, $\forall \boldsymbol{\omega} \in[02 \pi)^{d}$, the sum

$$
\begin{aligned}
& \sum_{\mathbf{k} \in \mathbb{Z}^{d}} \hat{h}(\boldsymbol{\omega}+2 \pi \mathbf{k}) \hat{\varphi}_{\text {opt }}(\boldsymbol{\omega}+2 \pi \mathbf{k}) \\
& \quad=\sum_{\mathbf{k} \in \mathbb{Z}^{d}}|\hat{h}(\boldsymbol{\omega}+2 \pi \mathbf{k})|^{2}|\hat{\beta}(\boldsymbol{\omega}+2 \pi \mathbf{k})|^{2} \\
& \quad>0
\end{aligned}
$$

is strictly positive so that $\varphi_{\text {opt }}$ satisfies the CS condition. For the converse implication, we see right away that the second SR condition is satisfied by $\varphi_{\text {opt }}$ because of (33) and Proposition 2. Then, from Remark 1 , we have $\left(h \star \varphi_{\text {opt }}\right)(\mathbf{k}) \in \ell_{1}\left(\mathbb{Z}^{d}\right)$. Therefore, the LHS of (34) is continuous and since it is also strictly positive, there exists $m>0$, such that $\sum_{\mathbf{k} \in \mathbb{Z}^{d}} \mid \hat{h}(\boldsymbol{\omega}+$ $2 \pi \mathbf{k})\left.\right|^{2}|\hat{\beta}(\boldsymbol{\omega}+2 \pi \mathbf{k})|^{4} \geq m>0$, which proves that the first $\mathbf{S R}$ condition is met as well.

The key to this equivalence is the optimality of $\varphi_{\text {opt }}(\mathbf{x})$ which leads to series of positive terms in both the $\mathbf{S R}$ and the $\mathbf{C S}$ conditions. However, the question still remains as how to ensure the existence of at least one nonvanishing product $\hat{h}\left(\boldsymbol{\omega}_{\mathbf{0}}+\right.$ $2 \pi \mathbf{k}) \hat{\beta}\left(\omega_{\mathbf{0}}+2 \pi \mathbf{k}\right)$ for each $\omega_{\mathbf{0}} \in[02 \pi)^{d}$ and some $\mathbf{k}=$ $\mathbf{k}\left(\boldsymbol{\omega}_{0}\right) \in \mathbb{Z}^{d}$. Unfortunately, this may be quite tedious to check directly. What we propose here is an easy alternative where the user only has to worry about the placement of the zeros of $\hat{L}(\boldsymbol{\omega})$ in relation to those of $\hat{h}(\boldsymbol{\omega})$.

Theorem 4: Let the following be true:

(i) L be spline-admissible;

(ii) $h(\mathbf{x}) \in L_{1}\left(\mathbb{R}^{d}\right)$ be nonpathological in the sense that there exists

$$
A_{h, 2}^{2}=\inf _{\boldsymbol{\omega} \in[02 \pi)^{d}} \sum_{\mathbf{k} \in \mathbb{Z}^{d}}|\hat{h}(\boldsymbol{\omega}+2 \pi \mathbf{k})|^{2}>0 ;
$$

(iii) $\hat{\mathrm{L}}(\boldsymbol{\omega})$ and $\hat{h}(\boldsymbol{\omega})$ have no common zeros.

Then, $\varphi_{\text {opt }}$ satisfies $\mathbf{S R}$ and $\mathbf{C S}$ conditions. 
Proof: First we note that $\hat{h}(\boldsymbol{\omega})$ and $\hat{\beta}(\boldsymbol{\omega})$ are continuous and bounded because $h(\mathbf{x}) \in L_{1}\left(\mathbb{R}^{d}\right)$ and $\mathrm{L}$ is spline-admissible. Suppose that $\hat{\mathrm{L}}\left(\boldsymbol{\omega}_{\mathbf{n}}\right)=0$ for $\boldsymbol{\omega}_{\mathbf{n}} \in \mathbb{R}, \mathbf{n} \in \mathcal{I}$ where $\mathcal{I} \subset \mathbb{Z}^{d}$ is a finite index set. These zeros must necessarily be cancelled by some corresponding zeros of $Q\left(e^{j \boldsymbol{\omega}}\right)$ which is $2 \pi$-periodic. This implies that

$$
\hat{\beta}\left(\boldsymbol{\omega}_{\mathbf{n}}+2 \pi \mathbf{k}\right)= \begin{cases}\mathcal{C}_{\beta, \mathbf{n}}, & \mathbf{k}=\mathbf{0} \\ \mathbf{0}, & \text { otherwise }\end{cases}
$$

where $\mathcal{C}_{\beta, \mathbf{n}} \neq 0$ is a real constant. Moreover, $\beta$ satisfies the SR condition because of the spline admissibility of L. Therefore, the only zeros of $\hat{\beta}(\boldsymbol{\omega})$ are $\left\{\boldsymbol{\omega}_{\mathbf{n}}+2 \pi \mathbf{k}, \mathbf{k} \in \mathbb{Z}^{d} \backslash\{\mathbf{0}\}\right.$, $\mathbf{n} \in \mathcal{I}\}$. Since $\hat{h}\left(\boldsymbol{\omega}_{\mathbf{n}}\right) \neq 0(\hat{\mathrm{L}}(\boldsymbol{\omega})$ and $\hat{h}(\boldsymbol{\omega})$ have no common zeros), we see that $\sum_{\mathbf{k} \in \mathbb{Z}^{d}}\left|\hat{h}\left(\boldsymbol{\omega}_{\mathbf{n}}+2 \pi \mathbf{k}\right)\right|^{2}\left|\hat{\beta}\left(\boldsymbol{\omega}_{\mathbf{n}}+2 \pi \mathbf{k}\right)\right|^{2}=$ $\left|\hat{h}\left(\boldsymbol{\omega}_{\mathbf{n}}\right)\right|^{2} \mathcal{C}_{\beta, \mathbf{n}}^{2}>0$, for all the zeros of $\hat{\beta}(\boldsymbol{\omega})$. Therefore, the only way $\hat{h}(\boldsymbol{\omega}+2 \pi \mathbf{k}) \hat{\beta}(\boldsymbol{\omega}+2 \pi \mathbf{k})$ can vanish is when $\hat{h}(\boldsymbol{\omega})$ has a $2 \pi$-periodic zero, which cannot be the case because of the lower Riesz bound. Thus, we are ensured that for each $\boldsymbol{\omega}_{0} \in\left[\begin{array}{ll}0 & 2 \pi\end{array}\right)^{d}$, there is at least one $\mathbf{k} \in \mathbb{Z}^{d}$ such that $\hat{h}\left(\boldsymbol{\omega}_{\mathbf{0}}+2 \pi \mathbf{k}\right) \hat{\beta}\left(\boldsymbol{\omega}_{\mathbf{0}}+2 \pi \mathbf{k}\right) \neq 0$. Following the argument used in Proposition 4, this implies that $\mathbf{S R}$ and $\mathbf{C S}$ conditions are both satisfied by $\varphi_{\text {opt }}(\mathbf{x})$.

The placement of the zeros $\hat{\mathrm{L}}(\boldsymbol{\omega})$ has some important effect on the reconstruction. Qualitatively, the regularization will be the least at the frequencies where $|\hat{\mathrm{L}}(\boldsymbol{\omega})|$ is minimum. In the limit when $\hat{\mathrm{L}}\left(\boldsymbol{\omega}_{\mathbf{n}}\right)=0$, the restoration filter simplifies to

$$
\begin{aligned}
R_{\lambda}\left(e^{j \boldsymbol{\omega}_{\mathbf{n}}}\right) & =\frac{1}{\sum_{\mathbf{k} \in \mathbb{Z}^{d}}\left|\hat{h}\left(\boldsymbol{\omega}_{\mathbf{n}}+2 \pi \mathbf{k}\right)\right|^{2}\left|\hat{\beta}\left(\boldsymbol{\omega}_{\mathbf{n}}+2 \pi \mathbf{k}\right)\right|^{2}} \\
& =\frac{1}{\hat{h}\left(\boldsymbol{\omega}_{\mathbf{n}}\right) \hat{\varphi}_{\mathrm{opt}}\left(\boldsymbol{\omega}_{\mathbf{n}}\right)}<\infty
\end{aligned}
$$

because $\left.Q\left(e^{j \boldsymbol{\omega}}\right)\right|_{\omega=\omega_{\mathbf{n}}}=0$ and $\hat{\beta}\left(\boldsymbol{\omega}_{\mathbf{n}}+2 \pi \mathbf{k}\right)=0, \mathbf{k} \in \mathbb{Z}^{d} \backslash\{\mathbf{0}\}$, by construction. Interestingly, this is the same response as that of a classical (nonregularized) inverse filter.

In light of this observation and Theorem 4, it makes good sense to place the zeroes (or minimal values) of $\hat{\mathrm{L}}(\boldsymbol{\omega})$, where $\hat{h}(\boldsymbol{\omega})$ takes its maximum (typically, $\boldsymbol{\omega}=0$ ) and vice versa. We will now consider some concrete examples and specify families of regularization operators that are well suited for low-pass systems. We will then return to the issue of the selection of the "best" regularization operator in Section VI, where we present an alternative stochastic formulation of the generalized sampling problem.

\section{CAse Illustrations}

In this section, we examine a few special cases of the generalized sampling setup. The key point is that the reconstruction space is derived from the regularization operator via the optimality condition OC. We illustrate this connection with concrete examples that are relevant to image processing.

\section{A. Ideal Versus Nonideal Sampling}

The prefilter $h(\mathbf{x})$ in the generalized sampling setup in Fig. 1 models the point spread function (PSF) of the acquisition device. The simplest, idealized case is $h(\mathbf{x})=\delta(\mathbf{x})$, which corresponds to a perfect sampling of the signal. The problem described by (19) then reduces to the multidimensional version of the smoothing spline problem investigated in previous work [35]-[37]. In that case, we can simply ignore $\hat{h}(\boldsymbol{\omega})$ in all formulas and invoke Theorem 4 to show that the problem is well posed, provided that the regularization operator $\mathrm{L}$ is spline-admissible.

Likewise, we can account for the physical effect of an optical system. In the paraxial approximation of optics, the system is shift-invariant with a general lowpass behavior; the optical PSF is in $L_{1}$ and does not exhibit structured set of zeros ( $2 \pi$-periodic). Hence, stability is usually not a problem.

By contrast, some man-made sensors, such as CCD cameras whose impulse responses are indicator functions, do exhibit zeroes on a regular grid in the Fourier domain; e.g., $h_{\mathrm{CCD}}\left(x_{1}, x_{2}\right)=\operatorname{rect}\left(x_{1}, x_{2}\right) \Rightarrow \hat{h}_{\mathrm{CCD}}(\boldsymbol{\omega})=0$, $\boldsymbol{\omega}=2 \pi \mathbf{k}, \mathbf{k} \neq \mathbf{0}$. Fortunately, the gain at zero frequency is nonzero (low-pass behavior) so that condition (35) is generally satisfied.

\section{B. Examples of Regularization Operators and Reconstruction Spaces}

Since most natural images are predominantly lowpass, it is desirable to reconstruct the lower part of the spectral content with minimum distortion which can be achieved through a judicious placement of the zeros of $\hat{\mathrm{L}}(\boldsymbol{\omega})$ near the origin [cf. (36)]. This strategy is also justified by the low-pass behavior of most PSFs and our desire to minimize instabilities by having $\hat{\mathrm{L}}(\boldsymbol{\omega})$ be small where $\hat{h}(\boldsymbol{\omega})$ is large, and vice versa. In what follows, we consider examples of multidimensional regularization operators that are associated with the most prominent families of spline functions: tensor-product polynomial splines [48]-[50], and polyharmonic splines [34], [51], the latter being the nonseparable counterpart of the former. We also introduce a generalized class of isotropic operators that lead to a new brand of "Matérn" splines, the relevance of which will be further justified in Section VI.

1) Separable Operators: We first study the separable case, where the Fourier transform of the multidimensional operator $\hat{\mathrm{L}}(\boldsymbol{\omega})$ can be decomposed into a product of simple monomials. The prototypical example is a succession of $n_{k}$-th order derivatives along each spatial coordinate leading to

$$
\hat{\mathrm{L}}_{\mathrm{Sep}}(\boldsymbol{\omega})=\prod_{k=1}^{d}\left(j \omega_{k}\right)^{n_{k}}, \quad n_{k} \geq 1 .
$$

Since $\hat{\mathrm{L}}_{\mathrm{Sep}}(\boldsymbol{\omega})$ has multiple zeros at $\boldsymbol{\omega}=\mathbf{0}$, we must choose $Q\left(e^{j \omega}\right)$ in (31) to cancel these out. The canonical choice is $Q_{\text {Sep }}\left(e^{j \omega}\right)=\prod_{k=1}^{d}\left(1-e^{j \omega_{k}}\right)^{n_{k}}$, which yields a multidimensional B-spline whose Fourier transform is

$$
\begin{aligned}
\hat{\beta}_{\mathrm{Sep}}(\boldsymbol{\omega}) & =\prod_{k=1}^{d}\left(\frac{1-e^{j \omega_{k}}}{j \omega_{k}}\right)^{n_{k}} \\
& =\prod_{k=1}^{d} e^{\frac{j \omega n_{k}}{2}}\left(\frac{2 \sin \left(\omega_{k} / 2\right)}{\omega_{k}}\right)^{n_{k}} .
\end{aligned}
$$


This expression is separable and can be inverted in a coordinatewise fashion. The end result is a tensor-product B-spline:

$$
\beta_{\mathrm{Sep}}(\mathbf{x})=\prod_{k=1}^{d} \beta^{n_{k}-1}\left(x_{k}+\frac{n_{k}}{2}\right)
$$

where $\beta^{n-1}(x)$ is Schoenberg's symmetric polynomial B-spline of degree $n-1$ (or order $n$ ) [49]. Since the 1-D B-splines are compactly supported and generate 1D Riesz bases, the same holds true for $\beta_{\text {Sep }}(\mathbf{x})$ in higher dimensions.

Let us now look at two concrete examples of sampling configurations. The first is $h(\mathbf{x})=\delta(\mathbf{x})$ (ideal sampling) with a second derivative regularization $n_{k}=2$ for all $k$. In that case, $\varphi_{\text {opt }}(\mathbf{x})=\left(\beta_{\text {Sep }} \star \bar{\beta}_{\text {Sep }}\right)(\mathbf{x})=\prod_{k=1}^{d} \beta^{3}\left(x_{k}\right)$, which is a tensor-product symmetrical cubic B-spline. Thus, we may rely on the present variational formalism to justify the use of cubic interpolation which is quite popular in image processing applications; this corresponds to the limiting case $\lambda \rightarrow 0$ where the data term vanishes (perfect fit).

The second example is $h(\mathbf{x})=\operatorname{rect}(\mathbf{x})$ (CCD camera) with a first derivative regularization $n_{k}=1$ for all $k$. In that case, $\varphi_{\text {opt }}(\mathbf{x})=\left(\bar{h} \star \beta_{\text {Sep }} \star \bar{\beta}_{\text {Sep }}\right)(\mathbf{x})=\prod_{k=1}^{d} \beta^{2}\left(x_{k}\right)$, which is a quadratic B-spline. Note that without the prefilter, we would have ended up with a linear spline solution which is the poor man's solution to the interpolation problem. As far as image analysis is concerned, we believe that a quadratic spline model is preferable, one of the reason being that it is better suited for the evaluation of image differentials because of its higher-order continuity [52]. Thanks to the present formulation and the fact that most images are captured using a pixel-integration device, we can invoke the present variational argument to support the use of quadratic splines in imaging applications. We are not aware of any previous justification in that direction.

2) Laplace Operators: The prime example of a nonseparable, isotropic operator is the $\gamma / 2$-iterated (or fractional) Laplacian whose Fourier transform is given by

$$
\hat{\mathrm{L}}_{\mathrm{Lap}}(\boldsymbol{\omega})=\|\boldsymbol{\omega}\|^{\gamma}, \quad \gamma>\frac{d}{2} .
$$

For $\gamma=2$, we recover the classical Laplacian which is a popular, local operator.

Similar to the 1-D separable case, the choice of an appropriate localization filter $Q_{\text {Lap }}\left(e^{j \omega}\right)=\|2 \sin (\boldsymbol{\omega} / 2)\|^{\gamma}$ that cancels out the multiple zeros at $\boldsymbol{\omega}=\mathbf{0}$ yields the polyharmonic B-spline function of order $\gamma$ for $\beta(\mathbf{x})$ [34], [53]; these can be written in the Fourier domain as

$$
\hat{\beta}_{\gamma}(\boldsymbol{\omega})=\frac{\left\|2 \sin \left(\frac{\omega}{2}\right)\right\|^{\gamma}}{\|\boldsymbol{\omega}\| \gamma}
$$

where $\|\boldsymbol{\omega}\|=\sqrt{\sum_{k=1}^{d} \omega_{k}^{2}}$ and $\|\sin (\boldsymbol{\omega})\|=\sqrt{\sum_{k=1}^{d} \sin ^{2}\left(\omega_{k}\right)}$. The polyharmonic B-splines fulfill the $\mathbf{S R}$ condition as well as the convolution property: $\beta_{\gamma_{1}} \star \beta_{\gamma_{2}}=\beta_{\gamma_{1}+\gamma_{2}}$.

If we now consider the case of an ideal sampling device together with a $\gamma / 2$-iterated Laplacian regularizer, we end up with the optimal generator $\varphi_{\text {opt }}(\mathbf{x})=\left(\beta_{\gamma} \star \bar{\beta}_{\gamma}\right)(\mathbf{x})=\beta_{2 \gamma}(\mathbf{x})$, which is a polyharmonic B-spline of order $2 \gamma$. The corresponding solution to the quadratic problem in Section III-D-5 is then the equivalent of the polyharmonic smoothing spline estimator investigated by Tirosh et al. [35].

3) Generalized Isotropic Operators: We now introduce a generalization of the fractional Laplacian operator that has the important advantage of being associated with an extended family of stationary processes, including the so-called Matérn class [54], [55]. This stochastic link will be made explicit in Section VI-B in connection with the MMSE (or Wiener) estimator. We note that there is also a stochastic interpretation of the polyharmonic spline estimator, but it is much more intricate because it involves fractional Brownian motion fields which are nonstationary processes [35], [36]. The idea here is to keep the isotropy property by having $\hat{\mathrm{L}}(\boldsymbol{\omega})$ be a function of $\|\boldsymbol{\omega}\|$, but to displace the zeros from the origin by considering an operator whose Fourier transform is

$$
\hat{\mathrm{L}}_{\mathrm{Iso}}(\boldsymbol{\omega})=\hat{\mathrm{L}}_{\mathrm{Iso}}(\|\boldsymbol{\omega}\|)=\prod_{m=1}^{N}\left(\alpha_{m}+\|\boldsymbol{\omega}\|^{2}\right)^{\gamma_{m}}
$$

with $\alpha_{m}>0, \gamma_{m}>(d / 2), m=1,2, \ldots, N$.

Since $\hat{\mathrm{L}}_{\mathrm{Iso}}(\boldsymbol{\omega})$ does not vanish, there is actually no need for localization. So we simply set $Q_{\mathrm{Iso}}\left(e^{j \omega}\right)=1$ and define the generalized Matérn B-spline with parameters $\left\{\left(\alpha_{m}, \gamma_{m}\right)\right\}_{m=1,2, \cdots, N}$ as $\hat{\beta}_{\text {Iso }}(\boldsymbol{\omega})=\prod_{m=1}^{N} \hat{\beta}_{\alpha_{m}, \gamma_{m}}(\boldsymbol{\omega})$, where $\hat{\beta}_{\alpha_{m}, \gamma_{m}}(\boldsymbol{\omega})=\left(\alpha_{m}+\|\boldsymbol{\omega}\|^{2}\right)^{-\gamma_{m}}$. Interestingly, we are able to compute the inverse Fourier transform of $\hat{\beta}_{\alpha_{m}, \gamma_{m}}(\boldsymbol{\omega})$ which is given by (see Appendix C)

$$
\beta_{\alpha_{m}, \gamma_{m}}(\mathbf{x})=\left(\frac{\|\mathbf{x}\|}{2 \sqrt{\alpha_{m}}}\right)^{\nu_{m}} \frac{\mathcal{K}_{\nu_{m}}\left(\sqrt{\alpha_{m}}\|\mathbf{x}\|\right)}{2^{d-1} \sqrt{\pi^{d}} \Gamma\left(\nu_{m}+\frac{d}{2}\right)}
$$

with $\nu_{m}=\gamma_{m}-d / 2 ; \mathcal{K}_{\nu}(u)$ is the modified Bessel function of the second kind which rapidly decays (faster than polynomial decay) for increasing $u$ and is positive for $\nu>0$ and $u>0$ [57]. This result constitutes the multidimensional extension of the 2-D formula given in [55].

Therefore, $\beta_{\alpha_{m}, \gamma_{m}}(\mathbf{x})$ is positive for $\gamma_{m}>d / 2$, and consequently $\beta_{\text {Iso }}(\mathbf{x})$, which is a $N$-fold convolution product

$$
\beta_{\text {Iso }}(\mathbf{x})=\beta_{\alpha_{1}, \gamma_{1}}(\mathbf{x}) \star \beta_{\alpha_{2}, \gamma_{2}}(\mathbf{x}) \star \ldots \beta_{\alpha_{N}, \gamma_{N}}(\mathbf{x})
$$

is positive as well. Moreover, since $\hat{\beta}_{\text {Iso }}(\boldsymbol{\omega}) \in L_{1}\left(\mathbb{R}^{d}\right), \beta_{\text {Iso }}(\mathbf{x})$ is uniformly continuous. The following proposition then ensures that $\hat{\mathrm{L}}_{\mathrm{Iso}}(\boldsymbol{\omega})$ is spline-admissible.

Proposition 5: For $\gamma_{m}>(d / 2), m=1,2, \ldots N$, the operator $\hat{\mathrm{L}}_{\mathrm{Iso}}(\boldsymbol{\omega})$ is spline-admissible, that is, the function $\beta_{\text {Iso }}(\mathbf{x})$ given by (44) satisfies the $\mathbf{S R}$ conditions.

Proof: Consider the periodic function $F(\boldsymbol{\omega})$ defined as

$$
\begin{aligned}
F(\boldsymbol{\omega}) & =\sum_{\mathbf{k} \in \mathbb{Z}^{d}}\left|\hat{\beta}_{\text {Iso }}(\boldsymbol{\omega}+2 \pi \mathbf{k})\right|^{2} \\
& =\sum_{\mathbf{k} \in \mathbb{Z}^{d}} \prod_{m=1}^{N} \frac{1}{\left(\alpha_{m}+\|\boldsymbol{\omega}+2 \pi \mathbf{k}\|^{2}\right)^{2 \gamma_{m}}} \\
& >\prod_{m=1}^{N} \frac{1}{\left(\alpha_{m}+4 \pi^{2} d\right)^{2 \gamma_{m}}}>0
\end{aligned}
$$

which involves strictly positive terms only. Thus, we have the lower Riesz bound inequality satisfied: $A_{\beta_{\text {Iso }, 2}} \geq \prod_{m=1}^{N}\left(\alpha_{m}+\right.$ 
$\left.4 \pi^{2} d\right)^{-\gamma_{m}}>0$. To prove the second $\mathbf{S R}$ condition, we observe that $\beta_{\text {Iso }}(\mathbf{x})>0, \forall \mathbf{x} \in \mathbb{R}$. Therefore, the task boils down to showing that $\sum_{\mathbf{k} \in \mathbb{Z}^{d}} \beta_{\text {Iso }}(\mathbf{x}+\mathbf{k})<+\infty, \forall \mathbf{x} \in[01)^{d}$. We first prove that this condition is satisfied by each $\beta_{\alpha_{m}, \gamma_{m}}(\mathbf{x})$. Consider the series $\sum_{\mathbf{n} \in \mathbb{Z}^{d}} \hat{\beta}_{\alpha_{m}, \gamma_{m}}(2 \pi \mathbf{n})$ which can be bounded from above

$$
\begin{aligned}
\sum_{\mathbf{n} \in \mathbb{Z}^{d}} \hat{\beta}_{\alpha_{m}, \gamma_{m}}(2 \pi \mathbf{n}) & =\sum_{\mathbf{n} \in \mathbb{Z}^{d}} \frac{1}{\left(\alpha_{m}+\|2 \pi \mathbf{n}\|^{2}\right)^{\gamma_{m}}} \\
& <\frac{1}{\alpha_{m}^{\gamma_{m}}}+\sum_{\mathbf{n} \in \mathbb{Z}^{d} \backslash\{\mathbf{0}\}} \frac{1}{\|2 \pi \mathbf{n}\|^{2 \gamma_{m}}}<+\infty
\end{aligned}
$$

where the right-hand side (RHS) series is known to converge whenever $\gamma_{m}>(d / 2)$. We then use Poisson's formula to obtain

$$
\begin{aligned}
\sum_{\mathbf{k} \in \mathbb{Z}^{d}} \beta_{\alpha_{m}, \gamma_{m}}(\mathbf{x}+\mathbf{k}) & =(2 \pi)^{d} \sum_{\mathbf{n} \in \mathbb{Z}^{d}} \hat{\beta}_{\alpha_{m}, \gamma_{m}}(2 \pi \mathbf{n}) e^{j 2 \pi \mathbf{n}^{\mathrm{T}} \mathbf{x}} \\
& \leq(2 \pi)^{d} \sum_{\mathbf{n} \in \mathbb{Z}^{d}} \hat{\beta}_{\alpha_{m}, \gamma_{m}}(2 \pi \mathbf{n})<+\infty .
\end{aligned}
$$

The result then follows from the repeated application of Proposition 2.

\section{STOCHASTIC FoRmULATIONS AND THE LINK WITH THE TIKHONOV SOLUTION}

The subject matter of the paper until now has been the theoretical analysis of a variational approach to the generalized sampling problem. The signal and measurements were treated as deterministic entities, and the solution to the problem was obtained by finding the global minimum of a cost functional. However, there are also alternative formulations for the situations where the measurements are of stochastic nature and where we have some a priori knowledge on the class of signals. The reconstruction problem can then be posed as an estimation problem where the continuous-space reconstruction is estimated from the given measurement such as to minimize the mean square error (MSE). Following [33] we consider two formulations. First the signal to be reconstructed is treated as deterministic, while the additive noise is considered to be of stochastic nature. In this case, we extend the results of [33] to multiple dimensions and perform a minimax estimation to obtain the reconstruction. In the second case, which we call the Wiener formulation, both the signal (continuous) and the noise (discrete) are modeled as stationary processes. The solution is obtained by minimizing the MSE of the reconstruction at each point in $\mathbb{R}^{d}$. Interestingly, this brings out a direct connection with the variational problem for the $p=2$ case (the Tikhonov criterion) and leads to identical reconstruction algorithms for some particular choice of $\mathrm{L}$ and $\lambda$.

\section{A. Connection With the Minimax Estimator}

When the signal is deterministic and the noise is stationary, the reconstruction problem can be posed as a minimax estimation problem [33]. In this case, we assume that the signal $s(\mathbf{x}) \in W_{L}^{2}$ with $\|\mathrm{L}\{s\}\|_{L_{2}} \leq \mu_{0}^{2}$ where the upper bound expresses our a priori knowledge on the class of input signals. The additive noise is modeled as a discrete, zero-mean stationary process with power spectral density (PSD) $C_{n}\left(e^{j \omega}\right)$.
The criterion we minimize is the worst case projected MSE which eliminates explicit dependence of the solution on the unknown signal [33]. The projection is made onto some reconstruction space $V_{\varphi}$ generated by $\varphi(\mathbf{x})$ which is decided a priori (and not necessarily optimal). In that framework, the minimization of the criterion over the signal coefficients $c[\mathbf{k}]$ yields a digital correction filter $r_{\mathrm{MX}}[\mathrm{k}]$ which, when applied on the measurements together with the basis function, gives the reconstruction, as illustrated in Fig. 2. Thus, our problem can be formulated as

$$
\arg \min _{s_{\mathrm{rec}} \in V_{\varphi},\left\|L\left\{s_{\mathrm{rec}}\right\}\right\|_{L_{2}} \leq \mu_{0}^{2}} E\left\{\left|s_{\mathrm{rec}}\left(\mathbf{x}_{0}\right)-s_{V_{\varphi}}\left(\mathbf{x}_{0}\right)\right|^{2}\right\}
$$

where $s_{V_{\varphi}}(\mathbf{x})$ is the orthogonal projection of the deterministic signal onto the shift-invariant space $V_{\varphi}$. The minimax reconstruction in $V_{\varphi}$ is then given by

$$
s_{\mathrm{rec}}(\mathbf{x})=\sum_{\mathbf{k} \in \mathbb{Z}^{d}}\left(r_{\mathrm{MX}} * g\right)[\mathbf{k}] \varphi(\mathbf{x}-\mathbf{k}) .
$$

By extending the argumentation of [33] to multiple dimensions, we can derive the frequency response of digital correction filter $r_{\mathrm{MX}}[\mathrm{k}]$ that minimizes the minimax criterion (45); it is given by

$$
\begin{aligned}
& R_{\mathrm{MX}}\left(e^{j \boldsymbol{\omega}}\right) \\
& =\frac{\left(\sum_{\mathbf{k} \in \mathbb{Z}^{d}} \frac{\hat{h}(\boldsymbol{\omega}+2 \pi \mathbf{k}) \hat{\varphi}(\boldsymbol{\omega}+2 \pi \mathbf{k})}{|\hat{\mathrm{L}}(\boldsymbol{\omega}+2 \pi \mathbf{k})|^{2}}\right)}{\left(\sum_{\mathbf{k} \in \mathbb{Z}^{d}}|\hat{\varphi}(\boldsymbol{\omega}+2 \pi \mathbf{k})|^{2}\right)\left(\frac{C_{n}\left(e^{j \boldsymbol{\omega}}\right)}{\mu_{0}^{2}}+\sum_{\mathbf{k} \in \mathbb{Z}^{d}} \frac{|\hat{h}(\boldsymbol{\omega}+2 \pi \mathbf{k})|^{2}}{|\hat{\mathrm{L}}(\boldsymbol{\omega}+2 \pi \mathbf{k})|^{2}}\right)} .
\end{aligned}
$$

The key point for our purpose is that, in the case of additive white noise, the minimax and Tikhonov reconstruction filters can be made rigorously equivalent, as stated in the following proposition.

Proposition 6: The reconstruction filters for the above minimax estimation problem and the Tikhonov problem in Section III-D-5 are identical provided that:

1) the discrete additive noise is white; i.e., $C_{n}\left(e^{j \omega}\right)=\sigma^{2}$;

2) the generator $\varphi(\mathbf{x})$ for the minimax method satisfies the optimality condition $\mathbf{O C}$; i.e., $\varphi(\mathbf{x})=\varphi_{\text {opt }}(\mathbf{x})$,

$3)$ the regularization parameter is set to its optimal value $\lambda_{\text {eq }}=\left(\sigma^{2} / \mu_{0}^{2}\right)$.

Proof: By substituting $C_{n}\left(e^{j \boldsymbol{\omega}}\right)=\sigma^{2}$ and $\hat{\varphi}(\boldsymbol{\omega})=$ $\hat{\varphi}_{\text {opt }}(\boldsymbol{\omega})=\hat{h}^{*}(\boldsymbol{\omega}) \hat{\phi}(\boldsymbol{\omega})$ where $\hat{\phi}(\boldsymbol{\omega})=\left(\left|Q\left(e^{j \boldsymbol{\omega}}\right)\right|^{2} /|\hat{\mathrm{L}}(\boldsymbol{\omega})|^{2}\right)$ and multiplying and dividing by $\left|Q\left(e^{j \omega}\right)\right|^{2}$ in the RHS of (47), we obtain the equation at the bottom of the next page. Thus, (46) is equivalent to (28) for $\lambda_{\mathrm{eq}}=\left(\sigma^{2} / \mu_{0}^{2}\right)$.

It is interesting to note that this equivalence only holds when we are considering the optimal generator $\varphi_{\text {opt }}$. Otherwise, the Tikhonov and minimax solution are generally different, as discussed in [33].

\section{B. Unification With the Wiener Formulation}

We now move one step further and consider that the input signal is a stochastic entity as well. Specifically, we assume that $s(\mathbf{x})$ is a realization of a continuous-space zero-mean stationary 
process with PSD $\hat{c}_{s}(\boldsymbol{\omega}) \in L_{2}\left(\mathbb{R}^{d}\right)$. The measurements are the same as in (13), except that the additive noise is now modeled as a zero-mean discrete stationary process whose autocorrelation and PSD are denoted by $c_{n}[\mathbf{k}]$ and $C_{n}\left(e^{j \omega}\right)$, respectively. It is further assumed that the signal and noise are uncorrelated.

The solution in this formulation is obtained by minimizing the MSE of the reconstruction at any fixed location $\mathbf{x}_{0} \in \mathbb{R}^{d}$

$$
\min E\left\{\left|s\left(\mathbf{x}_{0}\right)-s_{\text {rec }}\left(\mathbf{x}_{0}\right)\right|^{2}\right\} \text {. }
$$

It should be noted that we minimize the MSE of the reconstruction and not the projected MSE, as done in [33]. Hence, our solution is the global minimum of the MSE. Keeping in line with the previous reconstruction framework, we will show that the optimal solution belongs once again to an integer-shift-invariant space that is generated by some optimal function $\varphi_{\mathrm{W}}(\mathbf{x})$ where the subscript stands for Wiener.

We want to emphasize the fact that the minimization of (48) is a hybrid version (discrete input-continuous output) of the standard Wiener problem, which is usually either stated in the discrete or purely continuous domain [58]. This said, the method of proof and solution are quite similar to the traditional ones and have already been deployed in the context of generalized sampling [59]. Therefore, in what follows, we simply present the hybrid Wiener solution (without proof) in a form that suits our notation.

Proposition 7: Consider the measurements $G=\{g[\mathbf{k}]=$ $(h \star s)(\mathbf{k})+n[\mathbf{k}]\}_{\mathbf{k} \in \mathbb{Z}^{d}}$, where $s(\mathbf{x})$ is a realization of a continuous-space stationary process with autocorrelation function $c_{s}(\mathbf{x}) \in L_{2}\left(\mathbb{R}^{d}\right)$ and $n[\mathbf{k}]$ is a discrete stationary noise component with PSD $C_{n}\left(e^{j \boldsymbol{\omega}}\right)$. Then, the linear minimum mean square error (LMMSE) estimator of the signal $s\left(\mathbf{x}_{0}\right)$ given $G$ can be written as

$$
s_{\mathrm{rec}}\left(\mathbf{x}_{0}\right)=\sum_{\mathbf{k} \in \mathbb{Z}^{d}}\left(r_{\mathrm{W}} * g\right)[\mathbf{k}] \varphi_{\mathrm{W}}\left(\mathbf{x}_{\mathbf{0}}-\mathbf{k}\right)
$$

where the optimal Wiener generator is $\varphi_{\mathrm{W}}(\mathbf{x})=\left(\bar{h} \star c_{s}\right)(\mathbf{x})$, and where the frequency response of the optimal restoration (digital correction) filter $r_{\mathrm{W}}$ is given by

$$
R_{\mathrm{W}}\left(e^{j \boldsymbol{\omega}}\right)=\frac{1}{C_{n}\left(e^{j \boldsymbol{\omega}}\right)+\sum_{\mathbf{k} \in \mathbb{Z}^{d}} \hat{\varphi}_{\mathrm{W}}(\boldsymbol{\omega}+2 \pi \mathbf{k}) \hat{h}(\boldsymbol{\omega}+2 \pi \mathbf{k})} .
$$

It is important to note that this MMSE formula is valid for any $\mathbf{x}_{0}$ and that the complete estimator $s_{\text {rec }}(\mathbf{x}), \mathbf{x} \in \mathbb{R}^{d}$ is included in the integer-shift-invariant subspace space $V_{\varphi_{\mathrm{W}}}$. It is also well known from estimation theory that the Wiener solution is optimal among all estimators (not just among the linear ones) when both the signal and noise are Gaussian distributed.

We observe that the PSD of the measurements is given by $C_{g}\left(e^{j \boldsymbol{\omega}}\right)=\sum_{\mathbf{k} \in \mathbb{Z}^{d}} \hat{c}_{s}(\boldsymbol{\omega}+2 \pi \mathbf{k})|\hat{h}(\boldsymbol{\omega}+2 \pi \mathbf{k})|^{2}+C_{n}\left(e^{j \boldsymbol{\omega}}\right)$, which coincides with the denominator of (50). Thus, a necessary and sufficient condition for the Wiener filter to be well defined (i.e., bounded) is that this quantity be nonvanishing (a sufficient condition is $\left.C_{n}\left(e^{j \omega}\right)>0\right)$. Also, if we take the Fourier transform of (49), we see that the solution is of the same form as (30) where the Fourier transform of the equivalent basis function is given by

$$
\hat{\varphi}_{\mathrm{W}, \mathrm{eq}}(\boldsymbol{\omega})=\frac{\hat{\varphi}_{\mathrm{W}}(\boldsymbol{\omega})}{C_{g}\left(e^{j \boldsymbol{\omega}}\right)}=\frac{\hat{h}^{*}(\boldsymbol{\omega}) \hat{c}_{s}(\boldsymbol{\omega})}{C_{g}\left(e^{j \boldsymbol{\omega}}\right)} .
$$

Once again, the optimal reconstruction space is generally not bandlimited, unless either $h(\mathbf{x})$ or $s(\mathbf{x})$ are bandlimited to start with. Here too, the reconstruction filters for the deterministic and stochastic cases can be made equivalent. This equivalence helps us not only associate the optimal generating function to the autocorrelation function of the signal, but also allows us to choose the best regularization operator $\mathrm{L}$ and the best regularization parameter $\lambda_{\text {opt }}$ for the Tikhonov solution in Corollary 2 . Comparing the solutions (28) and (49) for the deterministic and stochastic cases respectively, we obtain the equivalence which corresponds to the case $Q\left(e^{j \omega}\right)=1$ and $C_{n}\left(e^{j \omega}\right)=\sigma^{2}$. That is, the Tikhonov and Wiener reconstruction algorithms become equivalent when the discrete additive noise is white and the regularization operator is chosen such that it whitens the input signal.

Proposition 8: The Tikhonov and Wiener solutions are functionally equivalent provided that:

1) $\mathrm{L}$ is the whitening operator of the underlying continuousspace stochastic process, i.e., $\mathrm{L}^{*} \mathrm{~L}\left\{c_{s}(\mathbf{x})\right\}=\sigma_{0}^{2} \delta(\mathbf{x})$

2) the measurement noise is white with variance $\sigma^{2}$;

3) the regularization parameter is set to its optimal value $\lambda_{\mathrm{opt}}=\left(\sigma^{2} / \sigma_{0}^{2}\right)$.

$$
\begin{aligned}
R_{\mathrm{MX}}\left(e^{j \boldsymbol{\omega}}\right)= & \left(\sum_{\mathbf{k} \in \mathbb{Z}^{d}} \hat{\varphi}_{\mathrm{opt}}(\boldsymbol{\omega}+2 \pi \mathbf{k}) \hat{h}(\boldsymbol{\omega}+2 \pi \mathbf{k}) \hat{\phi}(\boldsymbol{\omega}+2 \pi \mathbf{k})\right)\left(\sum_{\mathbf{k} \in \mathbb{Z}^{d}}\left|\hat{\varphi}_{\mathrm{opt}}(\boldsymbol{\omega}+2 \pi \mathbf{k})\right|^{2}\right)^{-1} \\
& \times\left(\frac{\sigma^{2}}{\mu_{0}^{2}}\left|Q\left(e^{j \boldsymbol{\omega}}\right)\right|^{2}+\sum_{\mathbf{k} \in \mathbb{Z}^{d}}|\hat{h}(\boldsymbol{\omega}+2 \pi \mathbf{k})|^{2} \hat{\phi}(\boldsymbol{\omega}+2 \pi \mathbf{k})\right)^{-1} \cdot \\
R_{\mathrm{MX}}\left(e^{j \boldsymbol{\omega}}\right)= & \frac{1}{\left(\lambda_{\mathrm{eq}}\left|Q\left(e^{j \boldsymbol{\omega}}\right)\right|^{2}+\sum_{\mathbf{k} \in \mathbb{Z}^{d}} \hat{h}(\boldsymbol{\omega}+2 \pi \mathbf{k}) \hat{\varphi}_{\mathrm{opt}}(\boldsymbol{\omega}+2 \pi \mathbf{k})\right)} \\
= & R_{\lambda_{\mathrm{eq}}}\left(e^{j \boldsymbol{\omega}}\right) .
\end{aligned}
$$


The proof is obvious once conditions 1) and 2) are substituted in (21) and (29).

We now conclude the section by illustrating this equivalence for the generalized isotropic operator introduced in Section V-B-3. Consider a stationary processes (for instance, the Matern process) whose PSD is given by $\hat{c}_{s}(\boldsymbol{\omega})=\sigma_{0}^{2}\left(\alpha+\|\boldsymbol{\omega}\|^{2}\right)^{-2 \gamma}$. It is readily seen that the corresponding whitening operator is $\hat{\mathrm{L}}_{\mathrm{Iso}}(\boldsymbol{\omega})=\left(\alpha+\|\boldsymbol{\omega}\|^{2}\right)^{\gamma}$, which is a special instance of (42) with $\gamma_{m}=\gamma$ and $N=1$. Therefore, the optimal estimate for such a process lies in the space generated by $\varphi_{\mathrm{W}}(\mathbf{x})=\left(\bar{h} \star \beta_{\text {Iso }} \star \bar{\beta}_{\text {Iso }}\right)(\mathbf{x})$, and the optimal regularization parameter is given by $\lambda_{\text {opt }}=\left(\sigma^{2} / \sigma_{0}^{2}\right)$. In the case where $\bar{h}(\mathbf{x})=\delta(\mathbf{x})$ (ideal sampling), the optimal generator happens to be a Matérn B-spline with parameters $(\alpha, 2 \gamma)$ whose explicit form is given by (43). Thus, the conclusion is that the Matérn splines are the optimal basis functions for the interpolation/approximation of such signals from their (noisy) samples.

Note that the equivalence established in the above corollary is meaningful only for operators of the type (42) and is not applicable to the Laplacian because the corresponding power spectral density is not defined in the classical sense.

\section{SUMMARY AND CONCLUSION}

We have considered the generalized sampling problem in multiple dimensions and addressed the issue of the "best" reconstruction space in the shift-invariant framework. We approached the problem from the interpolation point of view and formulated it as a generalized-smoothing spline problem where the continuous-space solution is obtained by the minimization of a data fidelity term subject to a continuous-space regularization constraint (Tikhonov-like functional) based on generalized differential operator L. In this formulation the signal and noise are treated as deterministic entities. By pure mathematical optimization, we showed that an optimal reconstruction space exists in the sense that the global minimum of our minimization problem belongs to this space, independently of the values of the measurements. An important point is that the optimal reconstruction space is shift-invariant in nature which justifies the use of this type of representation. Once an optimal shift-invariant basis is specified, the expansion coefficients of the solution can be obtained by means of a nonlinear optimization process. In the particular case where $p=2$ and $\Phi(x)=x$ (Tikhonov criterion), the solution has an explicit analytical form and can be computed by a one-step hybrid-linear filtering (discrete in-continuous out) of the measurements.

We have emphasized that optimality is achieved when the reconstruction space is "matched" to the chosen regularization operator. This was expressed in terms of an optimality condition that gives rise to a B-spline interpretation of $\varphi_{\mathrm{opt}}(\mathbf{x})$ and ensures the $L_{p}$-stability of the representation. The proposed formulation is quite general and extends most of the solutions to the sampling/interpolation problem that have been proposed so far; it also encompasses the various classes of smoothing spline estimators. We further justified this fact by presenting illustrations of various regularization operators and the corresponding reconstruction spaces.
Finally, we also presented a stochastic formulation of the generalized sampling problem, providing explicit minimax and MMSE solutions. We showed that, for appropriate regularization operator $\mathrm{L}$ and the regularization parameter $\lambda$, the solution corresponding to the deterministic Tikhonov criterion is functionally equivalent to one(s) obtained in the stochastic signal processing framework. The bottomline is that the regularization operator should be matched to the spectral behavior of the signal (whitening operator) and the regularization strength set inversely proportional to the signal-to-noise ratio (SNR).

\section{APPENDIX A PROOF OF THEOREM 1}

(a) Let us use the notation $\hat{f}_{\mathbf{k}}=\hat{f}(\boldsymbol{\omega}+2 \pi \mathbf{k})$ and $\hat{\mathrm{L}}_{\mathbf{k}}=$ $\hat{\mathrm{L}}(\boldsymbol{\omega}+2 \pi \mathbf{k})$. Consider $\mathcal{A}_{\mathcal{I}}(\boldsymbol{\omega})=\sum_{\mathbf{k} \in \mathcal{I}} \hat{f}_{\mathbf{k}}$ for some $f(\mathbf{x}) \epsilon$ $W_{L}^{2}$ and $\mathcal{I}$, a bounded subset of $\mathbb{Z}^{d}$. Using the Cauchy-Schwarz inequality, we have

$$
\begin{aligned}
\left|\mathcal{A}_{\mathcal{I}}(\boldsymbol{\omega})\right|^{2} & =\left|\sum_{\mathbf{k} \in \mathcal{I}} \frac{\sqrt{1+\left|\hat{\mathrm{L}}_{\mathbf{k}}\right|^{2}} \hat{f}_{\mathbf{k}}}{\sqrt{1+\left|\hat{\mathrm{L}}_{\mathbf{k}}\right|^{2}}}\right|^{2} \\
& \leq\left(\sum_{\mathbf{k} \in \mathcal{I}} \frac{1}{1+\left|\hat{\mathrm{L}}_{\mathbf{k}}\right|^{2}}\right)\left(\sum_{\mathbf{k} \in \mathcal{I}}\left(1+\left|\hat{\mathrm{L}}_{\mathbf{k}}\right|^{2}\right)\left|\hat{f}_{\mathbf{k}}\right|^{2}\right) \\
& \leq C_{0} \underbrace{\sum_{\mathbf{k} \in \mathbb{Z}^{d}}\left(1+\left|\hat{\mathrm{L}}_{\mathbf{k}}\right|^{2}\right)\left|\hat{f}_{\mathbf{k}}\right|^{2}}_{\mathcal{B}(\boldsymbol{\omega})} .
\end{aligned}
$$

But, $\mathcal{B}(\boldsymbol{\omega}) \in L_{2}\left([02 \pi)^{d}\right)$, as seen below: since $f(\mathbf{x}) \in W_{L}^{2}$ and using Fubini-Tonelli Theorem we write

$$
\begin{aligned}
\int_{[02 \pi)^{d}} \mathcal{B}(\boldsymbol{\omega}) d \boldsymbol{\omega} & =\sum_{\mathbf{k} \in \mathbb{Z}^{d}\left[02 \int_{2 \pi)^{d}}\right.}\left(1+\left|\hat{\mathrm{L}}_{\mathbf{k}}\right|^{2}\right)\left|\hat{f}_{\mathbf{k}}\right|^{2} d \boldsymbol{\omega} \\
& =\int_{\mathbb{R}^{d}}\left(1+|\hat{\mathrm{L}}(\boldsymbol{\omega})|^{2}\right)|\hat{f}(\boldsymbol{\omega})|^{2} d \boldsymbol{\omega} \\
& <+\infty .
\end{aligned}
$$

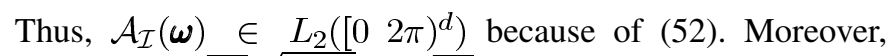
$\left|\mathcal{A}_{\mathcal{I}}(\boldsymbol{\omega})\right| \leq \sqrt{C_{0}} \sqrt{\mathcal{B}(\boldsymbol{\omega})} \leq \sqrt{C_{0}}(1+\mathcal{B}(\boldsymbol{\omega}))$, and

$$
\int_{[02 \pi)^{d}} \sqrt{\mathcal{B}(\omega)} d \boldsymbol{\omega} \leq(2 \pi)^{d}+\int_{[02 \pi)^{d}} \mathcal{B}(\boldsymbol{\omega}) d \boldsymbol{\omega}<+\infty .
$$

Now, consider the following limit: $\lim _{\mathcal{I} \rightarrow \mathbb{Z}^{d}} \mathcal{A}_{\mathcal{I}}(\boldsymbol{\omega})=\mathcal{A}(\boldsymbol{\omega})=$ $\sum_{\mathbf{k} \in \mathbb{Z}^{d}} \hat{f}_{\mathbf{k}}$. Since $\left|\mathcal{A}_{\mathcal{I}}(\boldsymbol{\omega})\right|^{2}$ is bounded by an integrable function [cf. (52)], we use Lebesgue's dominated convergence theorem and write

$$
\begin{aligned}
\int_{[02 \pi)^{d}}|\mathcal{A}(\boldsymbol{\omega})|^{2} d \boldsymbol{\omega} & =\int_{[02 \pi)^{d}} \lim _{\mathcal{I} \rightarrow \mathbb{Z}^{d}}\left|\mathcal{A}_{\mathcal{I}}(\boldsymbol{\omega})\right|^{2} d \boldsymbol{\omega} \\
& =\lim _{\mathcal{I} \rightarrow \mathbb{Z}^{d}} \int_{[02 \pi)^{d}}\left|\mathcal{A}_{\mathcal{I}}(\boldsymbol{\omega})\right|^{2} d \boldsymbol{\omega}<+\infty .
\end{aligned}
$$

Thus, $\mathcal{A}(\boldsymbol{\omega}) \in L_{2}\left(\left[\begin{array}{ll}0 & 2 \pi\end{array}\right)^{d}\right)$ and hence has a Fourier series expansion: $\mathcal{A}(\boldsymbol{\omega})=\sum_{\mathbf{k} \in \mathbb{Z}^{d}} c[\mathbf{k}] e^{-j \boldsymbol{\omega}^{\mathrm{T}} \mathbf{k}}$ a.e. Again, using 
Lebesgue's dominated convergence theorem $\left(\left|\mathcal{A}_{\mathcal{I}}(\boldsymbol{\omega})\right|\right.$ is bounded by an integrable function), the coefficient $c[\mathrm{k}]$ can be written as

$$
\begin{aligned}
c[\mathbf{k}] & =\frac{1}{(2 \pi)^{d}} \int_{[02 \pi)^{d}} \mathcal{A}(\boldsymbol{\omega}) e^{j \boldsymbol{\omega}^{\mathrm{T}} \mathbf{k}} d \boldsymbol{\omega} \\
& =\frac{1}{(2 \pi)^{d}} \int_{[02 \pi)^{d}} \lim _{\mathcal{I} \rightarrow \mathbb{Z}^{d}} \mathcal{A}_{\mathcal{I}}(\boldsymbol{\omega}) e^{j \boldsymbol{\omega}^{\mathrm{T}} \mathbf{k}} d \boldsymbol{\omega} \\
& =\frac{1}{(2 \pi)^{d}} \lim _{\mathcal{I} \rightarrow \mathbb{Z}^{d}} \int_{[02 \pi)^{d}} \mathcal{A}_{\mathcal{I}}(\boldsymbol{\omega}) e^{j \boldsymbol{\omega}^{\mathrm{T}} \mathbf{k}} d \boldsymbol{\omega} \\
& =\frac{1}{(2 \pi)^{d}} \int_{\mathbb{R}^{d}} \hat{f}(\boldsymbol{\omega}) e^{j \boldsymbol{\omega}^{\mathrm{T}} \mathbf{k}} d \boldsymbol{\omega}=f(\mathbf{k}) .
\end{aligned}
$$

Thus, the coefficients $c[\mathbf{k}]$ are nothing but the samples of the function $f(\mathbf{x})$ at $\mathbf{x}=\mathbf{k} \in \mathbb{Z}^{d}$. Finally, because

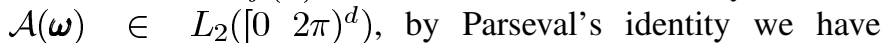
$f(\mathbf{k}) \in \ell_{2}\left(\mathbb{Z}^{d}\right)$.

\section{APPENDIX B}

\section{PROOF OF THEOREM 2}

For any $u \in W_{L}^{2}$, we have $(h \star u)(\mathbf{x}) \in W_{L}^{2}$ by Proposition 1 , as well as $(h \star u)(\mathbf{k}) \in \ell_{2}\left(\mathbb{Z}^{d}\right)$, by Theorem 1. Correspondingly, Proposition 3 ensures that $s_{\text {con }} \in W_{L}^{2}$.

Now, consider the function defined as $f(\mathbf{x})=u(\mathbf{x})-$ $s_{\text {con }}(\mathbf{x})$. Since $W_{L}^{2}$ is closed, $f(\mathbf{x}) \in W_{L}^{2}$. Consequently, $(h \star f)(\mathbf{x}) \in W_{L}^{2}$ and $(h \star f)(\mathbf{k}) \in \ell_{2}\left(\mathbb{Z}^{d}\right)$. But, $(h \star f)(\mathbf{k})=0, \forall \mathbf{k} \in \mathbb{Z}^{d}$, because of the consistency of $s_{\text {con }}(\mathbf{x})$. Therefore, the following Poisson summation formula holds: $\sum_{\mathbf{k} \in \mathbb{Z}^{d}}(h \star f)(\mathbf{k}) e^{-j \boldsymbol{\omega}^{\mathrm{T}} \mathbf{k}}=\sum_{\mathbf{k} \in \mathbb{Z}^{d}} \hat{h}(\boldsymbol{\omega}+2 \pi \mathbf{k}) \hat{f}(\boldsymbol{\omega}+2 \pi \mathbf{k})=$ 0 , a.e., and, hence

$$
\left\|\sum_{\mathbf{k} \in \mathbb{Z}^{d}} \hat{h}(\boldsymbol{\omega}+2 \pi \mathbf{k}) \hat{f}(\boldsymbol{\omega}+2 \pi \mathbf{k})\right\|_{L_{2}\left([02 \pi)^{d}\right)}=0 .
$$

Writing, $\|\mathrm{L} u\|_{L_{2}}^{2}=\left\|\mathrm{L}\left\{u-s_{\text {con }}+s_{\text {con }}\right\}\right\|_{L_{2}}^{2}=\|\mathrm{L}\{f\}\|_{L_{2}}^{2}+$ $\left\|\mathrm{L}\left\{s_{\text {con }}\right\}\right\|_{L_{2}}^{2}+\left\langle\mathrm{L}\{f\}, \mathrm{L}\left\{s_{\text {con }}\right\}\right\rangle_{L_{2}}$, it only remains to show that $\left\langle\mathrm{L}\{f\}, \mathrm{L}\left\{s_{\mathrm{con}}\right\}\right\rangle_{L_{2}}=0$.

Letting $D\left(e^{j \boldsymbol{\omega}}\right)=R_{0, \mathrm{opt}}\left(e^{j \boldsymbol{\omega}}\right)\left|Q\left(e^{j \boldsymbol{\omega}}\right)\right|^{2} \sum_{\mathbf{k} \in \mathbb{Z}^{d}} \hat{h}(\boldsymbol{\omega}+$ $2 \pi \mathbf{k}) \hat{u}(\boldsymbol{\omega}+2 \pi \mathbf{k})$, we see that

$$
\begin{aligned}
&\left\langle\mathrm{L}\left\{s_{\text {con }}\right\}, \mathrm{L}\{f\}\right\rangle_{L_{2}} \\
&= \frac{1}{(2 \pi)^{d}}\left\langle\hat{\mathrm{L}} \hat{s}_{\text {con }}, \hat{\mathrm{L}} \hat{f}\right\rangle_{L_{2}} \\
&= \frac{1}{(2 \pi)^{d}} \int_{\mathbb{R}^{d}} D\left(e^{j \boldsymbol{\omega}}\right) \hat{h}(\boldsymbol{\omega}) \hat{f}(\boldsymbol{\omega}) d \boldsymbol{\omega} . \\
&\left\langle\mathrm{L}\left\{s_{\mathrm{con}}\right\}, \mathrm{L}\{f\}\right\rangle_{L_{2}} \\
&=\lim _{\mathcal{I} \rightarrow \mathbb{Z}^{d}} \int_{[0} \frac{D\left(e^{j \boldsymbol{\omega}}\right)}{(2 \pi)^{d}}\left(\sum_{\mathbf{k} \in \mathcal{I}} \hat{h}(\boldsymbol{\omega}+2 \pi \mathbf{k}) \hat{f}(\boldsymbol{\omega}+2 \pi \mathbf{k})\right) d \boldsymbol{\omega} \\
& \leq \frac{1}{(2 \pi)^{d}}\left\|D\left(e^{j \boldsymbol{\omega}}\right)\right\|_{L_{2}\left([02 \pi)^{d}\right)}=0 . \\
& \quad \times \lim _{\mathcal{I} \rightarrow \mathbb{Z}^{d}}\left\|\sum_{\mathbf{k} \in \mathcal{I}} \hat{h}(\boldsymbol{\omega}+2 \pi \mathbf{k}) \hat{f}(\boldsymbol{\omega}+2 \pi \mathbf{k})\right\|_{L_{2}\left([02 \pi)^{d}\right)}=0
\end{aligned}
$$

Since $R_{0, \text { opt }}\left(e^{j \boldsymbol{\omega}}\right),\left|Q\left(e^{j \boldsymbol{\omega}}\right)\right|$ are bounded and $(h \star u)(\mathbf{k}) \in \ell_{2}$, the first norm is finite and by (55), the limit of the second norm tends to zero, thus, proving (24).

\section{APPENDIX C \\ INVERSE FOURIER TRANSFORM OF $\hat{\beta}_{\alpha_{m}, \gamma_{m}}(\boldsymbol{\omega})$}

Using the fact that

$$
\frac{1}{\left(\alpha_{m}+\|\boldsymbol{\omega}\|^{2}\right)^{\gamma_{m}}}=\frac{1}{\Gamma\left(\gamma_{m}\right)} \int_{0}^{\infty} t^{\gamma_{m}-1} e^{-t\left(\alpha_{m}+\|\boldsymbol{\omega}\|^{2}\right)} d t
$$

we write the inverse Fourier transform of $\hat{\beta}_{\alpha_{m}, \gamma_{m}}(\boldsymbol{\omega})$ as

$$
\begin{aligned}
\beta_{\alpha_{m}, \gamma_{m}}(\mathbf{x})=\frac{(2 \pi)^{-d}}{\Gamma\left(\gamma_{m}\right)} \int_{0}^{\infty} t^{\gamma_{m}-1} e^{-\alpha_{m} t+\frac{\|\mathbf{x}\|^{2}}{4 t}} & \times \underbrace{\left(\int_{\boldsymbol{\omega} \in \mathbb{R}^{d}} e^{-t\left(\left\|\boldsymbol{\omega}-j \frac{x}{2 t}\right\|^{2}\right)} d \boldsymbol{\omega}\right)}_{(\sqrt{\pi / t})^{d}} d t .
\end{aligned}
$$

Substituting $t=\left(\|\mathbf{x}\| / 2 \sqrt{\alpha_{m}}\right) e^{-u}$ and manipulating, we obtain

$$
\begin{aligned}
\beta_{\alpha_{m}, \gamma_{m}}(\mathbf{x}) & =\mathcal{C}\left(\frac{\|\mathbf{x}\|}{\sqrt{\alpha_{m}}}\right)^{\nu_{m}} \int_{0}^{\infty} \cosh \left(\nu_{m} u\right) e^{-\|\mathbf{x}\| \sqrt{\alpha_{m}} \cosh (u)} d u \\
& =\mathcal{C}\left(\frac{\|\mathbf{x}\|}{\sqrt{\alpha_{m}}}\right)^{\nu_{m}} \mathcal{K}_{\nu_{m}}\left(\sqrt{\alpha_{m}}\|\mathbf{x}\|\right)
\end{aligned}
$$

where $\nu_{m}=\gamma_{m}-(d / 2), \mathcal{C}=\left(\sqrt{(2 \pi)^{d}} 2^{\gamma_{m}-1} \Gamma\left(\gamma_{m}\right)\right)^{-1}$ and the integral in the last step is the modified Bessel function of the second kind [57].

\section{REFERENCES}

[1] C. E. Shannon, "Communication in the presence of noise," Proc. IRE, vol. 37, pp. 10-21, 1949.

[2] J. M. Whittaker, "The Fourier theory of the cardinal functions," Proc. Math. Soc. Edinburgh, vol. 1, pp. 169-176, 1929.

[3] D. A. Linden, "A discussion of sampling theorems," Proc. IRE, vol. 47, pp. 1219-1226, 1959.

[4] A. J. Jerri, "The Shannon sampling theorem-Its various extensions and applications: A tutorial review," Proc. IEEE, vol. 65, no. 11, pp. 1565-1596, 1977.

[5] M. Unser, "Sampling-50 years after Shannon," Proc. IEEE, vol. 88, no. 4, pp. $569-587,2000$.

[6] P. L. Butzer, "A survey of the Whittaker-Shannon sampling theorem and some of its extensions," J. Math. Res. Expo., vol. 3, pp. 185-212, 1983.

[7] E. T. Whittaker, "On the functions which are represented by the expansion of interpolating theory," Proc. R. Soc. Edinburgh, vol. 35, pp. 181-194, 1915.

[8] J. J. Benedetto, "Frames, sampling and seizure prediction," in Advances in Wavelets, K.-S. Lau, Ed. New York: Springer-Verlag, 1998, pp. $1-15$.

[9] J. R. Higgins, Sampling Theory in Fourier and Signal Analysis. Oxford, U.K.: Clarendon, 1996.

[10] D. P. Petersen and D. Middleton, "Sampling and reconstruction of wave-number-limited functions in $N$-dimensional Euclidean spaces," Inf. Control, vol. 5, pp. 279-323, 1962.

[11] J. L. Brown, Jr. and K. Sangsari, "Sampling reconstruction of $N$-dimensional band-limited images after multilinear filtering," IEEE Trans. Circuits Syst., vol. 36, no. 7, pp. 1035-1038, 1989. 
[12] A. Feuer and G. C. Goodwin, "Reconstruction of multidimensional bandlimited signals from nonuniform and generalized samples," IEEE Trans. Signal Process., vol. 53, no. 11, pp. 4273-4282, 2005.

[13] S. H. Izen, "Generalized sampling expansion on lattices," IEEE Trans. Signal Process., vol. 53, no. 6, pp. 1949-1963, 2005.

[14] H. R. Kunsch and F. A. Hamprecht, "Optimal lattices for sampling," IEEE Trans. Inf. Theory, vol. 51, no. 2, pp. 634-647, 2005.

[15] A. Papoulis, "Generalized sampling expansion," IEEE Trans. Circuits Syst., vol. 24, pp. 652-654, 1977.

[16] A. Feuer, "On the necessity of Papoulis' result for multidimensional GSE," IEEE Signal Process. Lett., vol. 11, no. 4, pp. 420-422, 2004.

[17] C. de Boor, R. A. Devore, and A. Ron, "Approximation from shiftinvariant subspaces of $L_{2}\left(\mathbb{R}^{d}\right)$," Trans. Amer. Math. Soc., vol. 341, no. 2, pp. 787-806, 1994.

[18] A. Aldroubi and M. Unser, "Sampling procedures in function spaces and asymptotic equivalence with Shannon's sampling theory," Numer. Funct. Anal. Optim., vol. 15, no. 1 \& 2, pp. 1-21, 1994.

[19] A. Aldroubi and K. Gröchenig, "Nonuniform sampling and reconstruction in shift invariant spaces," SIAM Rev., vol. 43, pp. 585-620, 2001.

[20] W. Chen, S. Itoh, and J. Shiki, "On sampling in shift-invariant spaces," IEEE Trans. Inf. Theory, vol. 48, no. 10, pp. 2802-2810, 2002.

[21] K. Yao, "Applications of reproducing kernel Hilbert spaces-Bandlimited signal models," Inf. Control, vol. 11, pp. 429-444, 1967.

[22] M. Z. Nashed and G. G. Walter, "General sampling theorems for functions in reproducing kernel Hilbert spaces," Math. Contr., Signals, Syst., vol. 4, pp. 373-412, 1991.

[23] Y. C. Eldar and T. Werther, "General framework for consistent sampling in Hilbert spaces," Int. J. Wavelets, Multiresolution Inf. Process., vol. 3, no. 4, pp. 497-509, 2005.

[24] Y. C. Eldar and T. G. Dvorkind, "A minimum squared-error framework for generalized sampling," IEEE Trans. Signal Process., vol. 54, no. 6, pp. 2155-2167, 2006.

[25] G. G. Walter, "A sampling theorem for wavelet subspaces," IEEE Trans. Inf. Theory, vol. 38, pp. 881-884, 1992.

[26] X. G. Xia and Z. Zhang, "On sampling theorem, wavelets, and wavelet transforms," IEEE Trans. Signal Process., vol. 41, pp. 3524-3535, 1993.

[27] I. Djokovic and P. P. Vaidyanathan, "Generalized sampling theorems in multiresolution subspaces," IEEE Trans. Signal Process., vol. 45, pp. 583-599, 1997.

[28] I. W. Selesnick, "Interpolating multiwavelets bases and the sampling theorem," IEEE Trans. Signal Process., vol. 47, no. 6, pp. 1615-1621, 1999.

[29] G. Demoment, "Image reconstruction and restoration-Overview of common estimation structures and problems," IEEE Trans. Acoust., Speech, Signal Process., vol. 37, no. 12, pp. 2024-2036, 1989.

[30] N. B. Karayiannis and A. N. Venetsanopoulos, "Regularization theory in image restoration-The stabilizing functional approach," IEEE Trans. Acoust., Speech, Signal Process., vol. 38, no. 7, pp. 1155-1179, 1990.

[31] M. R. Banham and A. K. Katsaggelos, "Digital image restoration," IEEE Signal Process. Mag., vol. 14, no. 2, pp. 24-41, 1997.

[32] M. Unser and A. Aldroubi, "A general sampling theory for nonideal acquisition devices," IEEE Trans. Signal Process., vol. 42, no. 11, pp. 2915-2925, 1994.

[33] Y. C. Eldar and M. Unser, "Non-ideal sampling and interpolation from noisy observations in shift-invariant spaces," IEEE Trans. Signal Process., vol. 54, no. 7, pp. 2636-2651, 2006.

[34] C. Rabut, "Elementary m-harmonic cardinal B-splines," Numer. Algorithms, vol. 2, pp. 39-62, 1992.

[35] S. Tirosh, D. Van De Ville, and M. Unser, "Polyharmonic smoothing splines and the multi-dimensional Wiener filtering of fractal-like signals," IEEE Trans. Image Process., vol. 15, no. 9, pp. 2616-2630, 2006.

[36] M. Unser and T. Blu, "Fractional splines and wavelets," SIAM Rev., vol. 42 , no. 1 , pp. $43-67,2000$.

[37] M. Unser and T. Blu, "Generalized smoothing splines and the optimal discretization of the Wiener filter," IEEE Trans. Signal Process., vol. 53, no. 6, pp. 2146-2159, 2005.

[38] S. Saitoh, V. K. Tuan, and M. Yamamotom, "Convolution inequalities and applications," J. Inequal. Pure Appl. Math., vol. 42, no. 3, 2003.

[39] H. H. Fu, M. Ng, M. Nikolova, and J. Barlow, "Efficient minimization methods of mixed $\ell_{2}-\ell_{1}$ and $\ell_{1}-\ell_{1}$ norms for image restoration," SIAM J. Scientif. Comput., vol. 27, no. 6, pp. 1881-1902, 2006.
[40] M. Nikolova, "Minimizers of cost-functions involving nonsmooth data-fidelity terms: Application to the processing of outliers," SIAM J. Numer. Anal., vol. 40, no. 3, pp. 965-994, 2002.

[41] S. Osher, L. Rudin, and E. Fatemi, "Nonlinear total variation based noise removal algorithms," Physica D, vol. 60, pp. 259-268, 1992.

[42] T. F. Chan and S. Esedoḡlu, "Aspects of total variation regularized $L^{1}$ function approximation," SIAM J. Appl. Math., vol. 65, no. 5, pp. 1817-1837, 2005.

[43] Y. Katznelson, An Introduction to Harmonic Analysis. New York: Dover, 1976.

[44] L. Schwartz, Théorie des distributions. Paris: Hermann, 1966.

[45] J. Duchon, "Splines minimizing rotation-invariant semi-norms in Sobolev spaces," in Multivariate Approximation Theory, W. Schempp and K. Zeller, Eds. Basel: Birkhäuser-Verlag, 1979, pp. 85-100.

[46] T. Blu and M. Unser, "Approximation error for quasi-interpolators and (multi-) wavelet expansions," Appl. Comp. Harm. Anal., vol. 6, no. 2, pp. 219-251, Mar. 1999.

[47] D. Pierre, Optimization Theory With Applications. New York: Dover, 1986.

[48] I. Schoenberg, "Contribution to the problem of approximation of equidistant data by analytic functions," Quart. Appl. Math., vol. 4, pp. 45-99, 1946, and 112-141.

[49] I. Schoenberg, "Cardinal interpolation and spline functions," J. Approx. Theory, vol. 2, pp. 167-206, 1969.

[50] I. Schoenberg, "Cardinal spline interpolation," CBMS-NSF Regional Conf. Ser. Appl. Math., vol. 12, 1973.

[51] W. R. Madych and S. A. Nelson, "Polyharmonic cardinal spline," $J$. Approx. Theory, vol. 60, pp. 141-156, 1990.

[52] P. Thévenaz and M. Unser, "Precision isosurface rendering of 3-D image data," IEEE Trans. Image Process., vol. 12, 2003.

[53] D. Van De Ville, T. Blu, and M. Unser, "Isotropic polyharmonic B-splines: Scaling functions and wavelets," IEEE Trans. Image Process., vol. 14, no. 11, pp. 1798-1813, 2005.

[54] M. L. Stein, Interpolation of Spatial Data-Some Theory for Kriging. New York: Springer-Verlag, 1999.

[55] B. Whitcher, "Wavelet-based bootstrapping of spatial patterns on a finite lattice," Computat. Statist. Data Anal., vol. 50, no. 9, pp. 2399-2421, 2006.

[56] B. Pesquet-Popescu and J. L. Véhel, "Stochastic fractal models for image processing," IEEE Signal Process. Mag., vol. 19, no. 5, pp. 48-62, 2002.

[57] M. Abramowitz and I. Stegun, Handbook of Mathematical Functions, 9th ed. New York: Dover, 1965.

[58] S. M. Kay, Statistical Signal Processing Vol. I: Estimation Theory. Englewood Cliffs, NJ: Prentice-Hall, 1995.

[59] C. L. Fales, F. O. Huck, J. A. McCormick, and S. K. Park, "Wiener restoration of sampled image data: End-to-end analysis," J. Opt. Soc. Amer. A, vol. 5, no. 3, pp. 300-314, 1988.

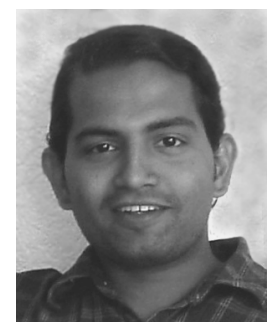

Sathish Ramani received the M.Sc. degree in physics from the Sri Sathya Sai Institute of Higher Learning (SSSIHL), Puttaparthy, Andhra Pradesh, India, in 2000 and the M.Sc. degree in electrical communication engineering from the Indian Institute of Science, Bangalore, in 2004.

Currently he is pursuing the Ph.D. degree at the Biomedical Imaging Group, Swiss Federal Institute of Technology (EPFL), Lausanne. His research interests include splines, interpolation, and variational methods in image processing.

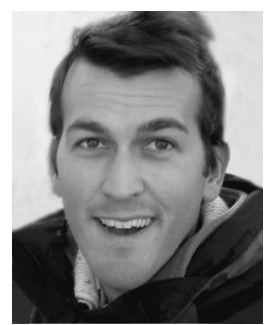

Dimitri Van De Ville (M'02) received the M.S. and $\mathrm{Ph} . \mathrm{D}$. degrees in engineering and computer sciences in 1998 and 2002, respectively, both from Ghent University, Belgium.

He then joined Prof. M. Unser's Biomedical Imaging Group, the Swiss Federal Institute of Technology (EPFL), Lausanne, where he continues his research. From December 2005, he become responsible for the Signal Processing Antenna at the University Hospital of Geneva as part of the Centre d'imagerie biomédicale (CIBM). His research 
interests include wavelets, statistical analysis, multidimensional splines, and applications in biomedical imaging.

Dr. Van De Ville serves as an Associate Editor for the IEEE TRANSACTIONS ON IMAGE PROCESSING (since February 2006), and was previously an Associate Editor for the IEEE Signal PROCESSING LeTTERS (2004-2006). Since 2003, he has also been Editor and webmaster of The Wavelet Digest, a noncommercial electronic newsletter for the wavelet community with more than 22000 subscribers. He organized the "Wavelets and Applications" semester of EPFL's Bernoulli Center, together with M. Vetterli and M. Unser, and WavE2006, its associated international conference (July 2006). He was also general co-chair of the Wavelets XII conference in San Diego, CA, held in August 2007.

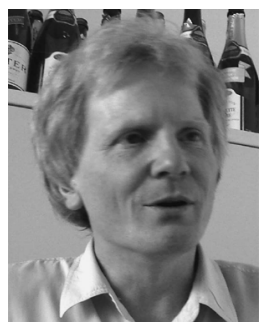

Thierry Blu (M'96-SM'06) was born in Orléans, France, in 1964. He received the "Diplôme d'ingénieur" from École Polytechnique, France, in 1986 and from Télécom Paris (ENST), France, in 1988. In 1996, he received the Ph.D. degree in electrical engineering from ENST for a study on iterated rational filterbanks, applied to wideband audio coding.

$\mathrm{He}$ is with the Biomedical Imaging Group, Swiss Federal Institute of Technology (EPFL), Lausanne, on leave from France Telecom R\&D Center, Issy-lesMoulineaux, France. At EPFL, he teaches the theory of Signals and Systems for Microengineering and Life Science. His research interests include (multi)wavelets, multiresolution analysis, multirate filterbanks, interpolation, approximation and sampling theory, image denoising, psychoacoustics, optics, and wave propagation.

Dr. Blu was the recipient of two Best Paper awards from the IEEE Signal Processing Society (2003 and 2006). Between 2002 and 2006, he has been an Associate Editor for the IEEE TRANSACTIONS ON IMAGE PROCESSING and since 2006, for the IEEE TRANSACTIONS ON Signal PROCESSING.

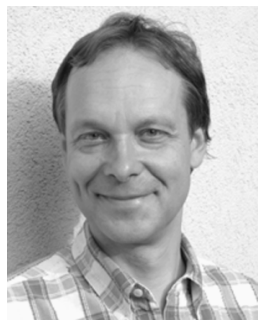

papers in these areas.

Dr. Unser has been actively involved with the IEEE TRANSACTIONS ON MEDICAL IMAGING, holding the positions of Associate Editor (1999-2002, 2006-present), a member of the Steering Committee, and Associate Editor-in-Chief (2003-2005). He has been an Associate Editor or member of the editorial board for eight more international journals, including the IEEE SIGNAL Processing Magazine, the IEEE TRANSACtions on IMAge Processing (1992-1995), and the IEEE Signal Processing LetTERs (1994-1998). He organized the first IEEE International Symposium on Biomedical Imaging (ISBI'2002). He currently chairs the Technical Committee of the IEEE-SP Society on Bio Imaging and Signal Processing (BISP), and well as the ISBI steering committee. He is the recipient of three Best Paper Awards from the IEEE Signal Processing Society. 